\title{
The nuclear properties and extended morphologies of powerful radio galaxies: the roles of host galaxy and environment
}

\author{
H. Miraghaei ${ }^{1}$, P. N. Best ${ }^{2}$ \\ ${ }^{1}$ School of Astronomy, Institute for Research in Fundamental Sciences, PO Box 19395-5531, Tehran, Iran \\ ${ }^{2}$ Institute for Astronomy (IfA), University of Edinburgh, Royal Observatory, Blackford Hill, EH9 3HJ Edinburgh, U.K.
}

\begin{abstract}
Powerful radio galaxies exist as either compact or extended sources, with the extended sources traditionally classified by their radio morphologies as Fanaroff-Riley (FR) type I and II sources. FRI/II and compact radio galaxies have also been classified by their optical spectra into two different types: high excitation (HERG; quasar-mode) and low excitation (LERG; jet-mode). We present a catalogue of visual morphologies for a complete sample of $>1000$ 1.4-GHz-selected extended radio sources from the Sloan Digital Sky Survey. We study the environment and host galaxy properties of FRI/II and compact sources, classified into HERG/LERG types, in order to separate and distinguish the factors that drive the radio morphological variations from those responsible for the spectral properties. Comparing FRI LERGs with FRII LERGs at fixed stellar mass and radio luminosity, we show that FRIs typically reside in richer environments and are hosted by smaller galaxies with higher mass surface density; this is consistent with extrinsic effects of jet disruption driving the FR dichotomy. Using matched samples of HERGs and LERGs, we show that HERG host galaxies are more frequently star-forming, with more evidence for disk-like structure than LERGs, in accordance with currently-favoured models of fundamentally different fuelling mechanisms. Comparing FRI/II LERGs with compact LERGs, we find the primary difference is that compact objects typically harbour less massive black holes. This suggests that lower-mass black holes may be less efficient at launching stable radio jets, or do so for shorter times. Finally, we investigate rarer sub-classes: wide-angle tail, head-tail, FR-hybrid and double-double sources.
\end{abstract}

Key words: galaxies: active - galaxies: interactions - radio continuum: galaxies

\section{INTRODUCTION}

Powerful radio galaxies display a very wide range of properties, both in their extended radio morphologies and in their optical spectra. Historically, the luminous radio galaxy population has been sub-divided in two different manners. Fanaroff \& Riley (1974) classified sources according to their radio morphologies as type I (FRI), in which the peak of radio emission is located near the core (edgedarkened), and type II (FRII), in which the peak of surface brightness is at the edge of the radio lobes far from the center of emission (edge-brightened). An alternative classification scheme is based on the relative intensity of high and low excitation lines in the optical spectrum (cf. Hine \& Longair 1979; Laing et al. 1994), comprising high-excitation radio galaxies (HERGs) and low-excitation radio galaxies (LERGs). The HERG and LERG populations are believed to represent intrinsically different types of objects (Best \& Heckman 2012). HERGs show high accretion rate (giving a total luminosity $>0.01 \mathrm{~L}_{\mathrm{Edd}}$, where $\mathrm{L}_{\mathrm{Edd}}$ is the Eddington luminosity) powered via accretion of cold gas, which may be brought in through mergers or interactions, or through secular processes such as non-axisymmetric perturbations or star forming winds. In contrast, LERGs show low accretion rates $\left(<0.01 \mathrm{~L}_{\mathrm{Edd}}\right)$ and are believed to be powered primarily via accretion of hot intergalactic gas (Heckman \& Best 2014; Yuan \& Narayan 2014). The host galaxies of the HERG and LERG populations are also different, with HERGs typically being hosted by galaxies of lower stellar mass, bluer colours, lower concentration (more disk-like) and lower black hole mass (Best \& Heckman 2012). The redshift evolution is also different for the two samples: HERGs show rapid cosmic evolution, while LERGs have little or no redshift evolution (Best et al. 2014; Pracy et al. 2016).

Two main descriptions have been proposed for the origin of the FR dichotomy. Early studies highlighted the different emission line properties of FRIs and FRIIs (Zirbel \& Baum 1995), and proposed that FRIs and FRIIs might be intrinsically different classes of objects, according to their central black hole parameters or jet content (Baum, Zirbel \& O’Dea 1995; Meliani, Keppens \& Sauty 2010). However, these emission line differences may be driven by the LERG/HERG classification, since in the samples studied there was a large overlap between the FRI and LERG populations, and 
FRIIs with HERGs. An alternative hypothesis is that the FR dichotomy is extrinsic, driven by the role of the host galaxy and surrounding environment. In this scenario, FRI and FRII radio galaxies are considered as fundamentally the same class of objects, with FRIs hosting less powerful jets which get disrupted by interactions in a dense surrounding environment (Kaiser \& Best 2007). In support of this model, several studies report an increased prevalence of FRIs in denser environment compared to FRIIs (Hill \& Lilly 1991; Gendre et al. 2010, 2013). In addition, the discovery of sources with hybrid morphologies, which are FRI on one side and FRII on the other side, strongly supports extrinsic models (GopalKrishna \& Wiita 2000; Gawronski et al. 2006; Ceglowski, Gawronski \& Kunert-Bajraszewska 2013). However the origin of the FR dichotomy might be related to a combination of environment and central engine properties (Wold, Lacy \& Armus 2007). The FR dichotomy has also been investigated through hydrodynamical and magneto-hydrodynamical simulations that have argued the jet disruptions can emerge from Kelvin-Helmholtz instabilities (Perucho et al. 2010), jet-stellar wind interactions (Wykes et al. 2015) or magnetic instabilities (Porth \& Komissarov 2015; Tchekhovskoy $\&$ bromberg 2015); these all favour the extrinsic scenario.

Studies of the evolution of the space density of FR radio galaxies over redshift (Clewley \& Jarvis 2004; Sadler et al. 2007; Rigby, Best \& Snellen 2008; Gendre et al. 2010), the host galaxy properties (Heckman et al. 1994; Baum et al. 1992, 1995; Govoni et al. 2000; Scarpa \& Urry 2001) and the black hole accretion mechanism (Gendre et al. 2013) have all helped us to understand the nature of FR dichotomy; however, it is still unclear what combination of intrinsic and extrinsic scenarios gives the most realistic description. A primary reason for this is because the extended morphologies of radio galaxies show a strong dependence on radio luminosity, and so does their HERG/LERG nature, and thus disentangling the two effects is challenging.

An example of this is the study of the cosmic evolution of the different radio source classes. It has been long-established that less powerful radio sources show less cosmic evolution than more powerful samples (e.g. Dunlop \& Peacock 1993 and references therein; Rigby et al. 2011). It has therefore been concluded that FRI sources with low radio powers show no redshift evolution (Clewley \& Jarvis 2004), while higher power FRI or FRII have rapid redshift evolution (Rigby, Best \& Snellen 2008; Gendre, Best \& Wall 2010). However, none of these previous studies took HERG/LERG classifications into account. More recent studies of the redshift evolution of HERG/LERG objects have shown that HERGs evolve very strongly and LERGs show little cosmic evolution, indicating that the luminosity dependence of the cosmic evolution might be driven by the changing relative contributions of HERG/LERG populations with luminosity (Best \& Heckman 2012; Best et al. 2014).

The motivation for the present study is to separate FRI/II dependencies from HERG/LERG dependencies, using four samples of FRI HERGs, FRI LERGs, FRII HERGs and FRII LERGs to investigate FRI/FRII and HERG/LERG properties independently. The host galaxy properties, together with environmental and galaxy interaction parameters will be investigated for all sub-samples.

Additionally, while FR radio galaxies have been identified by their extended morphologies, there is a class of compact radio sources which have no extended components in their radio structure. Baldi, Capetti \& Giovannini (2015) suggested a new type of radio galaxies called FR0, corresponding to compact sources in this study, which are more core dominated and display less extended radio emission compared to FRI/II radio galaxies. These compact radio galaxies dominate the population at lower radio luminosities (e.g. Best et al. 2005a). Studying compact radio sources can help us to determine whether these objects go through the same evolutionary path as the extended sources, and may shed light on the origin of different radio morphologies observed for radio galaxies. Previous studies have claimed that these compact sources may be: i) the same as the extended sources, but with radio jets and lobes viewed at a small angle to their axis (Blandford \& Königl 1979; Fanti et al. 1990); ii) young radio sources at the early stage of their evolution, which will later become FRI or FRII (Fanti et al. 1995); iii) shortlived radio galaxies, whose jets get disrupted due to the low jet bulk speed (hence unable to sustain extended radio jets), perhaps caused by a lower black hole spin (Baldi, Capetti \& Giovannini 2015) or interaction with the dense gas (O'Dea \& Baum 1997; Alexander 2000); iv) a fundamentally different class of objects which do not have potential of developing extended radio jets. In this study, we investigate the environment and host galaxy properties of compact radio sources, comparing them with the extended sources in order to examine the above scenarios.

The layout of our paper is as follows. The radio source samples and classifications are presented in Section 2. Results considering the overall differences of FRIs and FRIIs (irrespective of HERG/LERG classification) are shown in Section 3, and compared to the literature. Our main result, considering FRI/FRII, HERG/LERG and compact/extended comparisons using matched samples that remove other dependencies, are presented in Section 4. In Section 5 we discuss radio galaxies with special and complex morphologies, for which our classification produces significant samples. We summarise and draw conclusions in Section 6 Throughout the paper we assumed a $\Lambda C D M$ cosmology with the following parameters: $\Omega_{m}=0.3, \Omega_{\Lambda}=0.7$ and $H_{0}=100 h \mathrm{~km}$ $\mathrm{s}^{-1} \mathrm{Mpc}^{-1}$ where $h=0.70$.

\section{SAMPLE AND CLASSIFICATION}

In this section, we describe the selection criteria of FRI, FRII and compact sources, the method that we applied for FRI/FRII and HERG/LERG classifications and the host galaxy and environment properties of the sample that we use in this study.

\subsection{Sample selection; global constraints}

The radio source sample and the parent galaxy sample are taken from Best \& Heckman (2012), who have cross-matched the seventh data release (DR7; Abazajian et al. 2009) of the Sloan Digital Sky Survey (SDSS; York et al. 2000) with the National Radio Astronomy Observatory (NRAO) Very Large Array (VLA) Sky Survey (NVSS; Condon et al. 1998) and the Faint Images of the Radio Sky at Twenty centimetres (FIRST) survey (Becker, White \& Helfand 1995), following the techniques of Best et al. (2005a). We applied a lower redshift cut of $z>0.03$ due to the large angular size of the nearby sources (and potential errors in catalogued higher-level SDSS parameters) and considered objects only within the SDSS 'main galaxy' or 'luminous red galaxy' samples. A 40 mJy flux density cut was also applied so that there would be sufficient signalto-noise in any extended structures to allow morphological classification. Radio sources classified as having an AGN host (rather than having radio emission dominated by star formation; see Best \& Heckman 2012) were selected.

To investigate a sample of extended sources, and classify their morphologies, the sub-sample of sources with multiple components 


\begin{tabular}{|c|c|c|c|c|c|c|c|c|}
\hline $\begin{array}{r}\text { Plate } \\
\text { ID }\end{array}$ & $\begin{array}{r}\text { Julian } \\
\text { Date }\end{array}$ & $\begin{array}{r}\text { Fibre } \\
\text { ID }\end{array}$ & $\begin{array}{r}\text { RA } \\
\text { J2000 } \\
(\mathrm{h})\end{array}$ & $\begin{array}{r}\text { Dec. } \\
\text { J2000 } \\
\left({ }^{\circ}\right)\end{array}$ & $\mathrm{Z}$ & $\begin{array}{r}\log \left[\mathrm{L}_{\text {rad }, t}\right] \\
\left(\mathrm{W} \mathrm{Hz}^{-1}\right)\end{array}$ & $\begin{array}{r}\text { Size } \\
\text { in radio } \\
(\operatorname{arcsec}) \\
\end{array}$ & $\begin{array}{r}\mathrm{FR} \\
\text { class }\end{array}$ \\
\hline 267 & 51608 & 34 & 9.9446584 & -0.02334 & 0.1392 & 24.92 & 107.66 & 200 \\
\hline 267 & 51608 & 205 & 9.8472382 & -0.88775 & 0.2715 & 25.08 & 30.00 & 200 \\
\hline 267 & 51608 & 260 & 9.8285522 & -0.84008 & 0.0810 & 24.42 & 43.06 & 300 \\
\hline 269 & 51910 & 257 & 10.0313580 & -0.87805 & 0.1364 & 24.86 & 124.24 & 100 \\
\hline 271 & 51883 & 93 & 10.3095760 & -0.83961 & 0.3410 & 25.47 & 27.38 & 200 \\
\hline 273 & 51957 & 633 & 10.6016020 & 0.10189 & 0.0968 & 25.06 & 145.39 & 100 \\
\hline 274 & 51913 & 218 & 10.6525950 & -0.78773 & 0.0952 & 23.98 & 115.91 & 210 \\
\hline 275 & 51910 & 617 & 10.8205810 & 0.99589 & 0.1065 & 24.44 & 71.47 & 210 \\
\hline 276 & 51909 & 314 & 10.8225380 & -0.66806 & 0.0387 & 23.94 & 87.16 & 100 \\
\hline 276 & 51909 & 440 & 10.8330150 & 0.32231 & 0.0390 & 23.34 & 83.86 & 100 \\
\hline 279 & 51608 & 34 & 11.3555320 & -0.22246 & 0.1010 & 25.24 & 85.64 & 102 \\
\hline 284 & 51662 & 114 & 11.9204590 & -0.52610 & 0.1322 & 24.83 & 41.03 & 102 \\
\hline 285 & 51663 & 190 & 11.9948950 & -0.53164 & 0.1782 & 24.64 & 123.28 & 210 \\
\hline 286 & 51999 & 267 & 12.0303620 & -0.50945 & 0.3282 & 25.21 & 14.41 & 200 \\
\hline 287 & 52023 & 266 & 12.1842470 & -0.33479 & 0.3192 & 25.48 & 12.41 & 200 \\
\hline 287 & 52023 & 573 & 12.2428980 & 0.79107 & 0.2510 & 25.09 & 109.39 & 200 \\
\hline 288 & 52000 & 490 & 12.3237460 & 0.68112 & 0.4062 & 25.56 & 15.78 & 200 \\
\hline 288 & 52000 & 502 & 12.3424470 & 0.07151 & 0.1585 & 24.74 & 135.33 & 100 \\
\hline 290 & 51941 & 291 & 12.5461130 & -0.92355 & 0.2050 & 24.87 & 133.36 & 200 \\
\hline 291 & 51660 & 42 & 12.7461690 & -1.01928 & 0.1468 & 24.39 & 12.19 & 200 \\
\hline$\ldots$ & $\ldots$ & $\ldots$ & ... & $\ldots$ & $\ldots$ & $\ldots$ & $\ldots$ & $\ldots$ \\
\hline$\ldots$ & $\ldots$ & $\ldots$ & $\ldots$ & $\ldots$ & $\ldots$ & $\ldots$ & $\ldots$ & $\ldots$ \\
\hline
\end{tabular}

Table 1. Properties of the 1329 extended radio galaxies with $\mathrm{z}>0.03$. The first 20 sources are listed here: the full table is available electronically. Columns 1 to 3 are the SDSS identifications of the target sources. The next three columns are the coordinates and redshift of the sample objects. Column 7 is total radio luminosity. Column 8 is the size of the radio source in arcsec. Column 9 indicates the morphological classification of the radio source. This is expressed in three digits. The first (left-most) digit indicates the FR class: (1) represents FRI, (2) is for FRII, (3) for hybrid and (4) unclassifiable. The second (middle) digit indicates whether the FR classification is consider certain (0) or less secure (1). The third (right-most) digit highlights any special nature of the sources: (0) stands for normal, (1) for a double-double source, (2) for a wide-angle tailed source, (3) for diffuse, and (4) for head-tail radio galaxies. An example of each class is presented in Figure 1 as detailed in Table 2

\begin{tabular}{lrrrrrrll}
\hline Object & $\begin{array}{r}\text { Plate } \\
\text { ID }\end{array}$ & $\begin{array}{r}\text { Julian } \\
\text { Date }\end{array}$ & $\begin{array}{r}\text { Fibre } \\
\text { ID }\end{array}$ & $\begin{array}{r}\text { RA } \\
\text { J2000 } \\
(\mathrm{h})\end{array}$ & $\begin{array}{r}\text { Dec. } \\
\text { J2000 } \\
\left({ }^{\circ}\right)\end{array}$ & Z & $\begin{array}{r}\text { FR } \\
\text { class }\end{array}$ & Note \\
\hline A1 & 450 & 51908 & 38 & 9.2855509 & 55.15227 & 0.1820 & 100 & certain FRI \\
A2 & 2422 & 54096 & 67 & 8.4179606 & 12.73467 & 0.3216 & 200 & certain FRII \\
A3 & 759 & 52254 & 12 & 8.2815938 & 39.18779 & 0.4654 & 300 & FR hybrid \\
A4 & 904 & 52381 & 307 & 10.1744710 & 53.05367 & 0.3411 & 400 & unclassifiable \\
B1 & 596 & 52370 & 221 & 11.1525290 & 63.47019 & 0.4263 & 110 & uncertain FRI \\
B2 & 1202 & 52672 & 463 & 9.6429687 & 45.33995 & 0.4501 & 210 & uncertain FRII \\
B3 & 1724 & 53859 & 275 & 15.6371080 & 7.95388 & 0.3566 & 310 & uncertain FR hybrid \\
B4 & 1603 & 53119 & 165 & 11.0489000 & 11.25012 & 0.4747 & 400 & unclassifiable \\
C1 & 2750 & 54242 & 325 & 14.8007230 & 14.78097 & 0.2089 & 102 & wide-angle-tailed FRI \\
C2 & 796 & 52401 & 492 & 15.7547800 & 50.79831 & 0.4309 & 201 & double-double FRII \\
C3 & 1833 & 54561 & 586 & 15.3127010 & 6.23225 & 0.1021 & 104 & head-tail FRI \\
C4 & 814 & 52370 & 117 & 16.3080980 & 44.57584 & 0.1966 & 103 & diffuse FRI \\
\hline
\end{tabular}

Table 2. List of objects presented in Fig. 1 Column 1 represents the object label according to their rows and column in Fig. 1 Columns 2-4 are the SDSS identifications of the target sources. The next three columns are the coordinates and redshift of the objects. Column 8 is the FR class of the objects as described in Table 1 Column 9 is a note describing the type of the radio galaxies.

in either the FIRST or NVSS imaging (see Best et al. 2005a) were considered. Sources contained within a single FIRST component were not considered because it would be nearly impossible to judge their morphology.

\subsection{Morphological classification}

FRI/FRII Classification was primarily based upon the original definition of the two classes (Fanaroff \& Riley 1974), namely whether the distance between the peak of the emission on the opposite sides of the radio source was larger (FRII) or smaller (FRI) than half of the total size of the radio source. The extended radio sample were visually examined in order to morphologically classify them (cf. Best 2009). However, the relatively poor angular resolution of FIRST and the low sensitivity to extended low surface brightness structures limits the ability to determine both source sizes and peak locations, particularly for smaller sources. A degree of human interpretation was therefore required. An additional flag was therefore introduced to the classification, to note whether it was secure, or less certain. In a few cases a visual examination showed that the source had been incorrectly flagged as extended, and these were removed from the extended sample. In total, there were 1329 genuinely morphologically classified extended sources. 


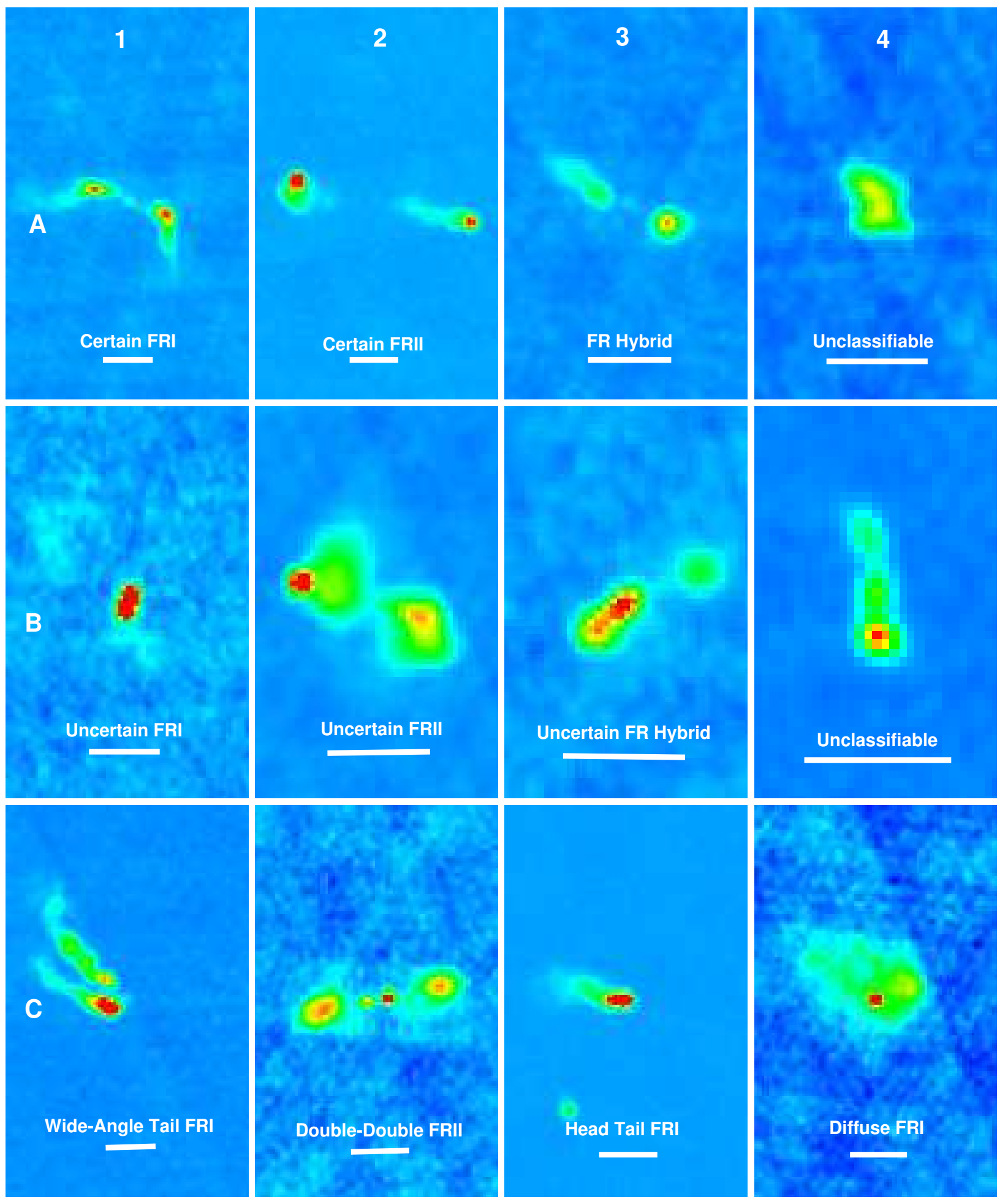

Figure 1. Examples of different classes of extended radio sources. The list of objects presented is given in Table 2 according to their row and column labels. The white bars are 30 arcsec length scale. For each source, the optical host galaxy position is precisely at the centre of the panel. 


\begin{tabular}{lrrr}
\hline & FRI & FRII & Compact \\
\hline HERG & 5 & 8 & 5 \\
LERG & 92 & 32 & 103 \\
Total & 97 & 40 & 108 \\
\hline
\end{tabular}

Table 3. Numbers of sources in the sample of galaxies $(z<0.1)$ with different classifications used in this study. 


\begin{tabular}{|c|c|c|c|c|c|c|c|c|c|c|c|c|c|c|c|c|c|c|c|c|}
\hline $\begin{array}{c}\text { Plate } \\
\text { ID }\end{array}$ & $\begin{array}{l}\text { Julian } \\
\text { Date }\end{array}$ & $\begin{array}{c}\text { Fibre } \\
\text { ID }\end{array}$ & $\begin{array}{r}\mathrm{RA} \\
\mathrm{J} 2000 \\
\text { (h) }\end{array}$ & $\begin{array}{r}\text { Dec. } \\
\mathrm{J} 2000 \\
\left({ }^{\circ}\right)\end{array}$ & $\bar{z}$ & $\mathrm{M}_{\star}$ & $\begin{array}{r}\mathrm{R}_{50} \\
(\mathrm{kpc})\end{array}$ & g-r & $\mathrm{D}_{4000}$ & $\mathrm{C}$ & $\mu_{50}$ & $\mathbf{M}_{B H}$ & $\eta$ & $\mathrm{Q}$ & $\mathrm{n}$ & PCA1 & PCA2 & $\mathrm{L}_{[\mathrm{OIII}]}$ & $\begin{array}{l}\text { HERG/ } \\
\text { LERG }\end{array}$ & $\begin{array}{l}\text { compact/ } \\
\text { FR1/FR2 }\end{array}$ \\
\hline 1016 & 52759 & 293 & 11.751812 & 53.64800 & 0.06901 & 11.46 & 7.04 & 1.01 & 2.00 & 3.22 & 8.96636 & 8.69 & 0.323 & -0.433 & 0.477 & 1.753 & 0.133 & 6.11 & 0 & 2 \\
\hline 1017 & 52706 & 284 & 11.836466 & 53.72242 & 0.06031 & 11.25 & 7.93 & 1.07 & 2.07 & 2.68 & 8.65305 & 8.38 & 0.510 & 0.874 & 0.602 & 2.709 & 0.724 & 5.97 & 0 & 2 \\
\hline 1044 & 52468 & 504 & 14.136240 & 52.68005 & 0.08287 & 11.63 & 12.15 & 1.06 & 1.94 & 2.58 & 8.66213 & 8.44 & 0.286 & -0.288 & 0.301 & 1.804 & 0.255 & 6.20 & 0 & 0 \\
\hline 1044 & 52468 & 602 & 14.197066 & 52.81670 & 0.07649 & 11.43 & 6.33 & 0.94 & 1.70 & 3.42 & 9.02853 & 8.61 & 0.466 & -0.805 & 0.903 & 1.673 & -0.226 & 6.37 & 0 & 0 \\
\hline 1046 & 52460 & 612 & 14.589354 & 50.85637 & 0.09969 & 11.22 & 6.65 & 1.08 & 1.64 & 3.09 & 8.77514 & 7.98 & -1.512 & -0.763 & 0.301 & -0.231 & 1.727 & 6.41 & 0 & 0 \\
\hline 1169 & 52753 & 172 & 15.998331 & 44.70899 & 0.04173 & 11.04 & 3.54 & 1.07 & 1.89 & 3.26 & 9.14332 & 7.96 & -0.447 & -1.296 & 0.477 & 0.493 & 0.372 & 6.23 & 0 & 0 \\
\hline 1170 & 52756 & 473 & 16.134591 & 43.16347 & 0.08490 & 11.49 & 7.61 & 1.04 & 2.03 & 2.97 & 8.92854 & 8.41 & 0.418 & 1.100 & 0.477 & 2.753 & 0.948 & 6.15 & 0 & 1 \\
\hline 1176 & 52791 & 637 & 16.983614 & 32.49423 & 0.06274 & 11.22 & 4.42 & 1.04 & 2.00 & 3.24 & 9.13068 & 8.53 & 0.715 & -1.119 & 0.845 & 1.729 & -0.655 & 6.33 & 0 & 1 \\
\hline 1184 & 52641 & 415 & 8.313819 & 4.10876 & 0.09462 & 11.21 & 5.02 & 1.01 & 1.85 & 3.18 & 9.01026 & 8.40 & 0.265 & -2.108 & 0.477 & 0.706 & -0.801 & 6.17 & 0 & 0 \\
\hline 1186 & 52646 & 613 & 8.615519 & 5.54502 & 0.09927 & 11.30 & 8.74 & 0.99 & 1.64 & 2.42 & 8.61879 & 7.66 & -1.358 & -1.156 & 0.301 & -0.313 & 1.344 & 7.23 & 1 & 2 \\
\hline 1192 & 52649 & 448 & 9.049780 & 6.32909 & 0.07708 & 11.31 & 6.55 & 1.01 & 2.03 & 3.07 & 8.87890 & 8.37 & -0.621 & -1.512 & 0.301 & 0.195 & 0.415 & 5.99 & 0 & 2 \\
\hline 1198 & 52669 & 611 & 9.021818 & 40.11377 & 0.09617 & 11.12 & 5.71 & 1.01 & 1.91 & 3.58 & 8.80738 & 8.27 & -0.176 & -1.066 & 0.699 & 0.893 & 0.245 & 6.15 & 0 & 1 \\
\hline 1220 & 52723 & 37 & 11.104728 & 8.97591 & 0.08502 & 11.35 & 7.83 & 1.04 & 2.04 & 3.15 & 8.76413 & 8.51 & -1.339 & -0.684 & 0.477 & -0.015 & 1.605 & 6.21 & 0 & 1 \\
\hline 1222 & 52763 & 423 & 11.288224 & 9.95881 & 0.07891 & 11.07 & 3.95 & 1.02 & 1.92 & 3.22 & 9.07829 & 8.51 & -0.794 & -0.945 & 0.477 & 0.362 & 0.919 & 6.19 & 0 & 0 \\
\hline 1237 & 52762 & 433 & 10.201777 & 8.69253 & 0.09844 & 11.32 & 8.25 & 1.04 & 2.02 & 3.24 & 8.68821 & 8.41 & 0.621 & -0.564 & 0.602 & 1.967 & -0.235 & 6.38 & 0 & 1 \\
\hline 1238 & 52761 & 296 & 10.320063 & 8.07998 & 0.08654 & 11.32 & 8.55 & 1.04 & 2.06 & 3.21 & 8.65719 & 8.19 & -1.120 & -1.674 & 0.477 & -0.387 & 0.805 & 6.15 & 0 & 1 \\
\hline 1238 & 52761 & 550 & 10.389618 & 8.86699 & 0.06260 & 11.22 & 3.72 & 1.03 & 2.05 & 3.56 & 9.27952 & 8.60 & -1.143 & -0.692 & 0.477 & 0.172 & 1.408 & 6.09 & 0 & 1 \\
\hline 1265 & 52705 & 158 & 8.093057 & 24.16399 & 0.05968 & 10.99 & 3.27 & 0.96 & 1.68 & 3.26 & 9.16118 & 8.17 & -0.065 & -3.100 & 0.699 & -0.202 & -1.067 & 7.79 & 1 & 2 \\
\hline 1269 & 52937 & 228 & 8.667324 & 29.81740 & 0.06484 & 11.24 & 9.28 & 0.95 & 1.42 & 2.51 & 8.50579 & 8.26 & -1.381 & 0.538 & 0.000 & 0.667 & 2.368 & 7.78 & 1 & 2 \\
\hline 1272 & 52989 & 114 & 9.126363 & 32.95634 & 0.04906 & 11.04 & 6.96 & 1.12 & 1.49 & 2.41 & 8.55564 & 7.74 & -0.486 & -1.767 & 0.000 & 0.176 & 0.133 & 5.71 & 0 & 0 \\
\hline$\ldots$ & $\ldots$ & $\ldots$ & $\ldots$ & $\ldots$ & $\ldots$ & $\ldots$ & $\ldots$ & $\ldots$ & $\ldots$ & $\ldots$ & $\ldots$ & $\ldots$ & $\ldots$ & $\ldots$ & $\ldots$ & $\ldots$ & $\ldots$ & $\ldots$ & $\ldots$ & $\ldots$ \\
\hline$\ldots$ & $\ldots$ & $\ldots$ & $\ldots$ & $\ldots$ & $\ldots$ & $\ldots$ & $\ldots$ & $\ldots$ & $\ldots$ & $\ldots$ & $\ldots$ & $\ldots$ & $\ldots$ & $\ldots$ & $\ldots$ & $\ldots$ & $\ldots$ & $\ldots$ & $\ldots$ & $\ldots$ \\
\hline
\end{tabular}

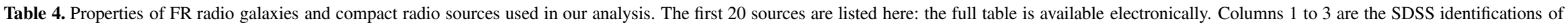

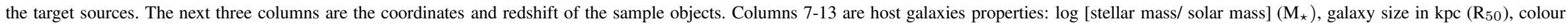

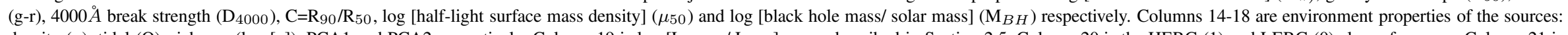

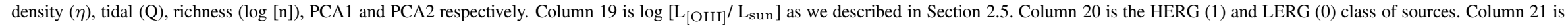
the compact ( 0 ) and extended ( 1 for FRI and 2 for FRII) labels for target sources. 


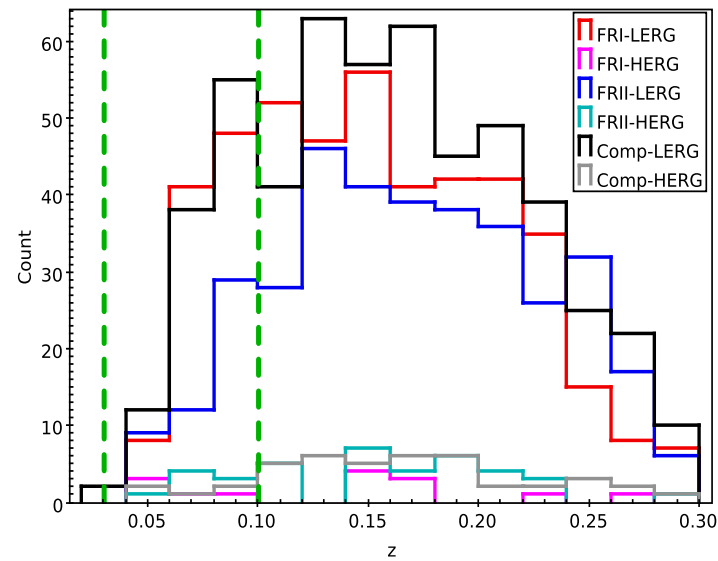

Figure 2. The redshift distribution of FR radio galaxies and compact radio AGN, separated into HERG and LERG classifications. The vertical lines are upper and lower redshift limit cuts applied to the all samples for the analysis in this paper.

Some sources presented morphologies which did not fit obviously into an FRI or FRII morphology. 35 sources were classified as hybrid sources (Gopal-Krishna \& Wiita 2000), which display an FRI-like morphology on one side of the nucleus and an FRIIlike morphology on the opposite side. A further 40 sources were deemed to be unclassifiable. Additionally some sources presented interesting morphologies which were given additional flags. These are 5 double-double sources (Schoenmakers et al. 2000), 9 head-tail sources (Rudnick \& Owen 1976) and 53 wide-angle tailed sources (cf. Owen \& Rudnick 1976). Examples of each of these sources are shown in Figure 1. The source classifications for the full sample of sources are given in Table 1

In addition to classifying the morphology, the visual analysis confirmed the NVSS and FIRST components of which the sources were comprised, allowing total fluxes (and hence luminosities) and radio source sizes to be determined. These properties are also provided in Table 1 Total radio fluxes were obtained by summing across the NVSS component fluxes; these should be reliable for sources with sizes upto $\approx 500$ arcsec (the largest angular size observable in snapshot observations with the VLA at $1.4 \mathrm{GHz}$ in Darray configuration), but may be underestimated for sources larger than this size (of which there are only 3 ). Source sizes are determined from the maximum angular separation of the centroids of the catalogued NVSS and/or FIRST components of the source. These may marginally underestimate the sizes of poorly resolved sources, but should provided a good approximation. Furthermore, the sizes of FRI sources are likely to be underestimated, since the emission from these gets progressively fainter with distance from the nucleus, and the most extended emission is likely to be missed by the short observations of NVSS and FIRST.

\subsection{Selection of subsamples for analysis}

For the goal of this paper, additional constraints have also been applied to the sample. We applied an upper redshift cut of $z<0.1$ due to the completeness limits in spectroscopic classification of HERGs and LERGs (see Section 2.4), and in finding companion galaxies for environmental studies (see Section 2.5). The redshift distribution of FRI and FRII radio sources are shown in Fig.2 FRI sources have, on average, lower redshifts than FRIIs, as expected in a fluxlimited sample since they are typically found at lower luminosities.

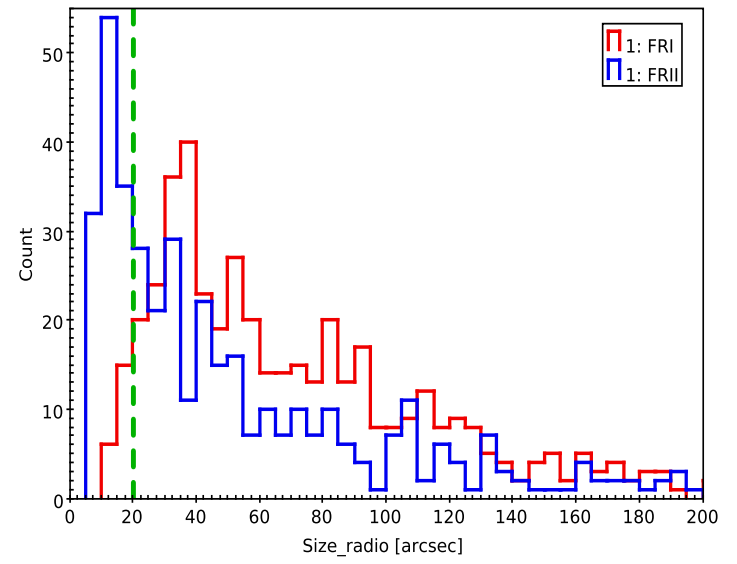

Figure 3. The distribution of radio sizes of the FR radio galaxies. The vertical line is the lower limit cut we applied to FRI and FRII samples for the analysis in this paper.

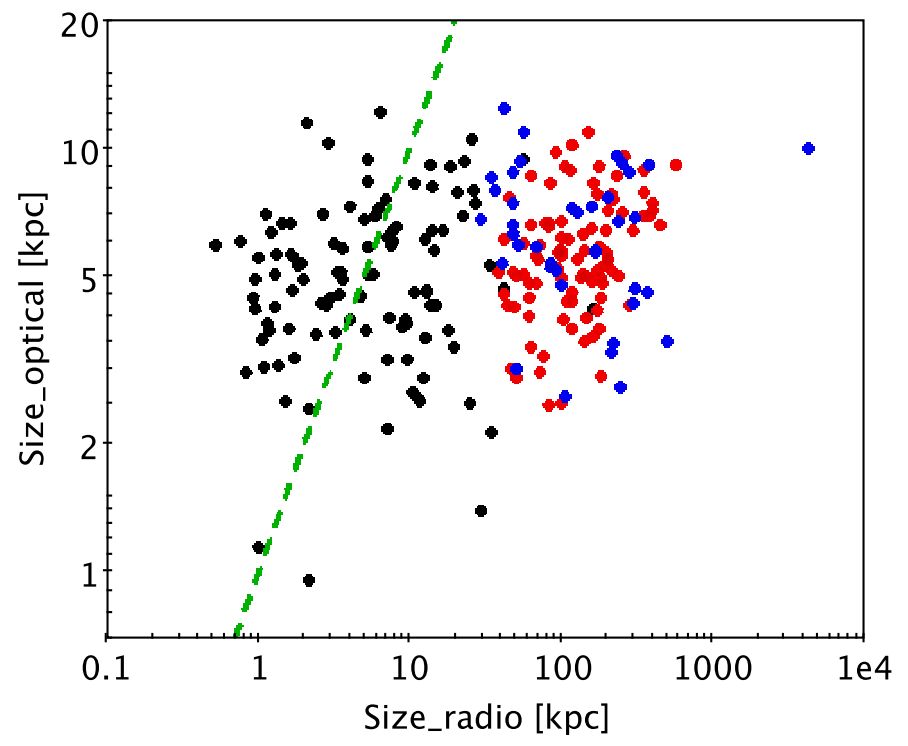

Figure 4. The radio and optical sizes (in kpc) of the compact (black), FRI (red) and FRII (blue) sources. The diagonal line represents equality between the both scales.

The distribution of angular sizes of FRI/FRII populations are shown in Fig. 3 It is notable that there is a dearth of FRI sources with angular sizes below 20 arcsec. This is believed to be due to the biases in classification of the sources arising from the low angular resolution and low surface brightness sensitivity of FIRST. Small-scale FRI sources, especially those whose extended emission is faint, may well be catalogued by FIRST as a single (albeit extended) component, and thus excluded from our analysis (which was restricted to multi-component sources; see Section 2.1). To avoid these biases in our further analysis, we hereafter restrict both FRI and FRII samples to the objects with angular sizes above $20^{\prime \prime}$. The final sample size for each classification is presented in Table 3.

For comparison with the extended sample, we also define a sample of compact radio sources. These correspond to those sources identified as single-component FIRST sources in the Best \& Heckman (2012) sample, with the same additional constraints: i) objects only within the SDSS 'main galaxy' or 'luminous red galaxy' samples, ii) with the redshift cuts of $0.03<\mathrm{z}<0.1$, iii) 
and the flux density cut of $\mathrm{S}>40 \mathrm{mJy}$. The redshift distribution of compact sources are also shown in Fig. 2

The optical sizes of the host galaxies compared to the radio sizes (in $\mathrm{kpc}$ ) of the compact and extended radio sources are displayed in Fig. 4 The diagonal line represents equality between the two scales (though, note these are differently defined, as the radio size is the full size of the sources whereas the optical size is a half-light radius). Compact radio sources are distributed around the equality line while extended sources are at the larger radio sizes. Therefore, the FRI and FRII sources typically extend well beyond their host galaxies and are large enough to be affected by the surrounding environment as well as conditions within their host galaxy which are both subjects of this study. We will consider both the extended and compact samples in Section 4.

\subsection{HERGS/LERGS classification}

The method used to classify sources into the two class of HERG and LERG has been extensively described by Best \& Heckman (2012) who considered the ratios of four high excitation lines ([OIII], [NII], [SII] and [OI]) to the $\mathrm{H}_{\alpha}$ and $\mathrm{H}_{\beta}$ emission lines, and also the equivalent width of the [OIII] emission line. They used the line-ratio diagnostic diagrams from Kewley et al. (2006) and also Cid Fernandes et al. (2011). We adopt the source classifications derived by Best $\&$ Heckman. Note that these were only complete out to $z=0.1$, which is one reason why this was adopted as an upper redshift limit in our analysis. The radio sample including HERG/LERG classifications is presented in Table 4

Using these classifications together with the morphological classifications from Section 2.2, the sample has been divided into six sub-samples of FRI HERGs, FRI LERGs, FRII HERGs, FRII LERGs, compact HERGs and compact LERGs with which we can study how the environment and host galaxy properties relate separately to the HERG/LERG and FRI/FRII/compact classifications. The sample size for each classification is presented in Table 3 while Fig. 2] shows the redshift distributions of the six sub-samples. The LERG sources are dominant in the sub-samples of FRI, FRII and compact, although the fraction of HERGs is highest for the FRIIs. Since there are relatively few sources classified as HERGs, when considering the FRI/II comparison in the rest of the paper we focus only on the FR LERGs.

\subsection{Host galaxy and environment properties of the sample}

Host galaxy properties for the radio sources are extracted from the value-added spectroscopic catalogues produced by the group from the Max Planck Institute for Astrophysics, and Johns Hopkins University (cf. Brinchmann et al. 2004). In particular, the parameters used in this paper are defined and estimated as follow:

stellar mass $\left(M_{\star}\right.$ or Mass) is derived from the extinctioncorrected optical luminosity using the mass to light ratio (Kauffmann, Heckman \& White 2003).

black hole mass $\left(\mathrm{M}_{B H}\right)$ is estimated using the velocity dispersion $\left(\sigma_{\star}\right)$ of the galaxy and the relation between the velocity dispersion and the black hole mass given in Tremaine et al. (2002): $\log \left(\mathrm{M}_{B H} / \mathrm{M}_{\star}\right)=8.13+4.02 \log \left(\sigma_{\star} / 200 \mathrm{~km} \mathrm{~s}^{-1}\right)$.

absolute magnitude $\left(\mathrm{M}_{r}\right)$ is the SDSS r-band absolute magnitude.

size which is $\mathrm{R}_{50}$, defined by the radius containing 50 percent of the galaxy light in the r-band. half-light surface mass density $\left(\mu_{50}\right)$ which is calculated using the relation: $\mu_{50}=0.5 \mathrm{M}_{\star} /\left(\pi \mathrm{R}_{50}{ }^{2}\right)$.

concentration $(C)$ calculated from the relation: $C=R_{90} / R_{50}$, where $\mathrm{R}_{90}$ is the radius containing 90 percent of the r-band galaxy light. Galaxies with high concentration index $(C>2.6)$ are typically bulge-dominated systems whereas galaxies with $\mathrm{C}<2.6$ are mostly disk-dominated systems (see Kauffmann et al. 2003).

colour $(\mathrm{g}-\mathrm{r})$ at rest-frame.

$4000 \AA$ break $\left(D_{4000}\right)$ which is strength of the $4000 \AA$ break of the galaxy optical spectrum, and is small for young stellar populations and large for old, metal-rich galaxies, thus giving a guide to the age of the galaxy.

OIII luminosity which is calculated from the detected [OIII] 5007 emission line provided this line is detected with a $\mathrm{S} / \mathrm{N}$ ratio above 2.5. In the case of no detection, we have used the corresponding upper limit luminosity of the 2.5 sigma flux density.

total radio luminosity $\left(\mathrm{L}_{\mathrm{rad}, t}\right)$ is calculated from the total radio flux obtained by summing across the NVSS component fluxes.

core radio luminosity $\left(\mathrm{L}_{\mathrm{rad}, \mathrm{c}}\right)$ is calculated from the radio flux of the central FIRST component of the galaxy.

To obtain the environment and galaxy interaction parameters we cross-matched the main catalogue with the environmental catalogue from Sabater, Best \& Argudo-Fernandez (2013). They defined and estimated three interacting parameters of density, tidal force (hereafter tidal) and richness, as follows:

density $(\eta)$ which is defined from the distance $\left(r_{10}\right.$ in Mpc) to the 10th nearest neighbour, $\eta=\log \left[10 /\left(4 \pi r_{10}^{3} / 3\right)\right]$.

tidal interaction (Q) which is defined by the relative tidal forces exerted by companions (i) with respect to the internal binding forces of the target galaxy (t). Here, $R$ is the radius of the target galaxy, $d$ is the distance between the target and the companion, and $L r$ is the corrected luminosity of the galaxy in $r$ band.

$$
Q_{t}=\log \left[\sum_{i}\left(L r_{t} / L r_{i}\right)\left(2 R_{t} / d_{i, t}\right)^{3}\right]
$$

richness (n) is the number of galaxies in the cluster or group to which the target galaxy belongs, as derived from the friends-offriends catalogue of Tago et al. (2010).

Sabater et al. (2013) also carried out a principal component analysis, to combine the density and tidal parameters in a way that removes the observed correlation between these two parameters. They thus introduced two new parameters:

PCA1 which traces the overall interaction level and environmental density of a galaxy.

PCA2 in which a higher value traces higher one-on-one interactions and a lower value traces galaxies that are relatively isolated for their overall environment.

The host galaxy and environment properties of the samples are listed in Table 4

\section{OVERALL PROPERTIES OF THE SAMPLES}

The FRI/II and HERG/LERG dichotomies have been explored extensively in the literature for both the host galaxy properties and the environment. In this section, we look at the overall properties of both classifications and compare our results with the previous studies. The sample selection has been described in Section 2 and the results are presented in Figs. 5- 8 . The left panel of Fig. 5 

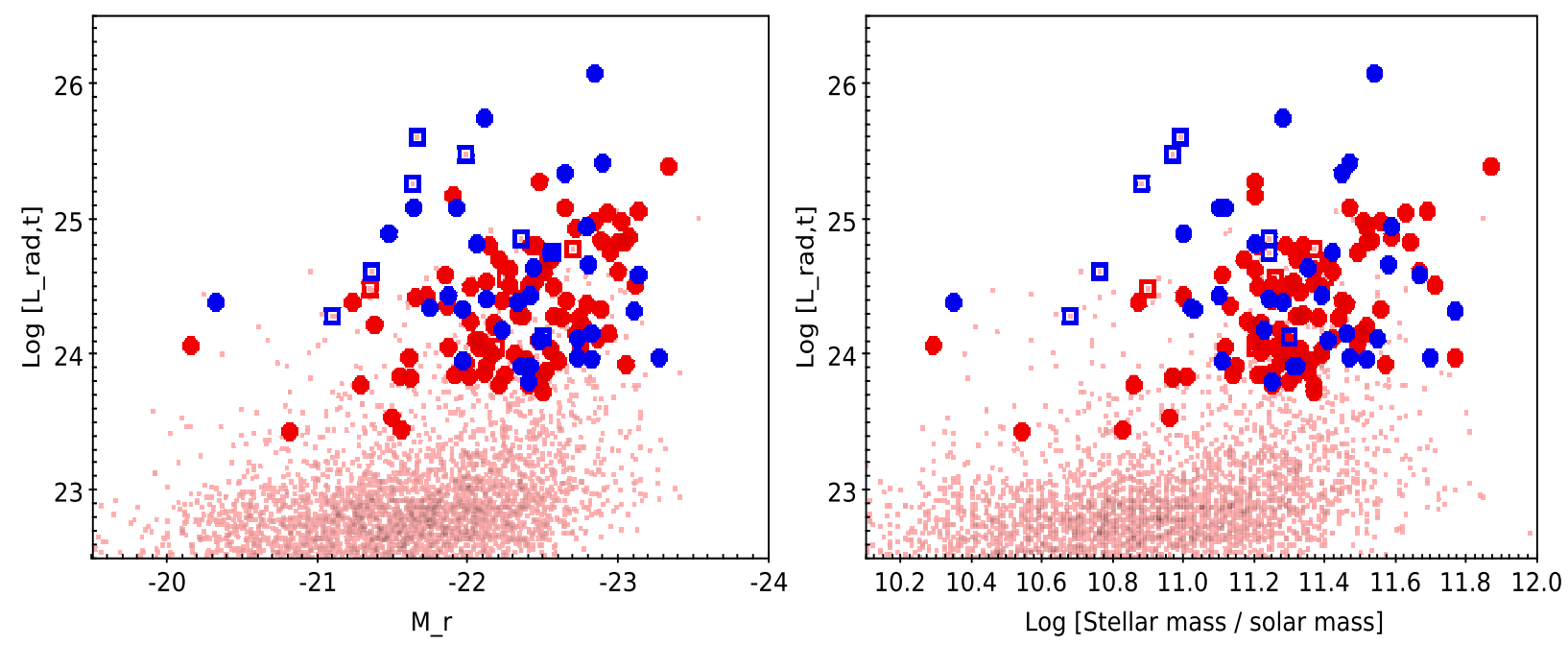

Figure 5. Total radio luminosity of the FRI (red) and FRII (blue) radio galaxies versus r-band absolute magnitude (left panel) and stellar mass (right panel). The filled circles represent LERGs and open squares are HERGs. Pink circles are all galaxies (hereafter galaxies with $0.03<\mathbf{z}<0.1$ ).

shows how FR galaxies are distributed in $\mathrm{L}_{r a d, t}-\mathrm{M}_{r}$ plane. Although there is a tendency for the high radio luminosity objects to be FRIIs, the sharp division line between the two class of objects that has been previously reported by Ledlow \& Owen (1996) is not seen; this result was also found by Best (2009). We observe the same distribution in $\mathrm{L}_{r a d, t}-\mathrm{M}_{\star}$ plane (Fig. 5 right panel). Since there is a tight correlation between stellar mass and optical absolute magnitude of the galaxies, we use stellar mass and total radio luminosity as the main parameters in the rest of the paper.

Fig. 6. displays the host galaxy properties of the radio galaxies, compared to the underlying galaxy population. The radio galaxy hosts lie at the tip of the $\mathbf{M}_{\star}-\mathbf{M}_{B H}$ distribution (Fig. 6 top-left), as expected since the most massive galaxies are more likely to host a radio-loud AGN (Best et al. 2005b). They also follow the stellar mass-black hole mass correlation line. The top-right panel of Fig. 6 shows the same behaviour for the $R_{50}$ versus $M_{\star}$ relation: the radio galaxies reside along the upper mass envelope of the galaxy population but with a comparable size distribution to underlying galaxies of the same mass. The concentration $\left(\mathrm{R}_{90} / \mathrm{R}_{50}\right)$ and half-light surface mass density $\left(\mu_{50}\right)$ versus stellar mass diagrams (Fig. 6 middle-left and middle-right) illustrate a clear tail of radio galaxies away from the main distribution of the galaxy population. This is also seen in the $4000 \AA$ break distribution, and to a lesser extent the g-r colour, with a scatter of sources towards bluer colours and younger stellar ages respectively (Fig. 6, bottom-right and bottomleft).

Fig. 7 compares the environmental parameters of the radio galaxies with the full galaxy population. It can be seen that the radio galaxies are typically distributed towards higher density (Fig.7 , top-left), tidal (Fig. 7 top-right) and PCA1 (Fig. 7 lower-left) parameters compared to other galaxies of the same mass. On the other hand, there is no significant offset between the radio galaxies and the underlying population in the PCA2 (one-on-one interactions) parameter (Fig. 7]lower-right).

Concentrating on the properties of the two FR samples, the FRIs and FRIIs (red $v s$ blue points) have broadly similar distributions in mass while FRIIs tend to have lower black hole masses. In contrast, Wold et al. (2007) argue that FRI and FRII have the same black hole mass distribution, but they also argue that for FRIs with low-excitation spectra the black hole masses correlates with radio luminosity, so sample selection limits might explain this difference. The important point is that the black-hole to stellar mass ratio appears lower for FRIIs than FRIs. No remarkable differences are observed in the distribution of sizes of the host galaxies $\left(\mathrm{R}_{50}\right)$. FRIIs tend to have lower concentration and lower $\mu_{50}$ than FRIs, and a larger proportion of the FRIIs than FRIs lie within the tails towards lower colour and lower $4000 \AA$ break. In a similar study, Raimann et al. (2005) showed that the stellar populations of FR I galaxies are, on average, older than those of FRIIs. Comparing the environments of FRIs with FRIIs, on average FRIIs clearly reside in lower density environments than FRIs, and are affected by slightly lower tidal forces. They also have lower PCA1 that confirms they are typically in lower density regions. Both samples show similar PCA2 distributions, indicating that the small differences in tidal forces might be a projection effect associated with the denser environments of FRIs. These result are all consistent with the previous studies that claim FRI radio galaxies are in denser environment (e.g. Prestage and Peacock 1988, Hill and Lilly 1991, Gendre et al 2013). Finally, FRIs have also brighter cores in radio, which is expected as this is part of their definition (Fig. 8, left panel).

As the plots show, many of these differences might also have emerged from a study of the HERG/LERG dichotomy, as clear differences are also seen between HERG and LERG objects at those parameters. For instance, HERGs appear to have higher total radio luminosity, lower black hole mass, bluer colours, and reside in lower density environments than LERGs (Figs 5. 8). The result for the black hole mass has been previously reported by Best \& Heckman (2012) while both higher (Smith \& Heckman 1989) and lower (Gendre et al. 2013) galaxy interaction have been reported for the HERG sources. It is noticeable that in some properties, the HERG/LERG separation appears to be a stronger driving factor than FRI/II differences: in particular, it is predominantly the HERG population (both FRI and FRII) which have weaker $4000 \AA$ breaks and bluer colours than typical galaxies of their stellar mass. Therefore, a lot of observed differences between FRIs and FRIIs may be caused by the HERG/LERG nature of the FR sources, and this issue has caused lots of misunderstanding and confusion in the study of FR radio galaxies when HERG/LERG classification is not taken into account.

In order to obtain a clean picture of FRI/FRII differences 

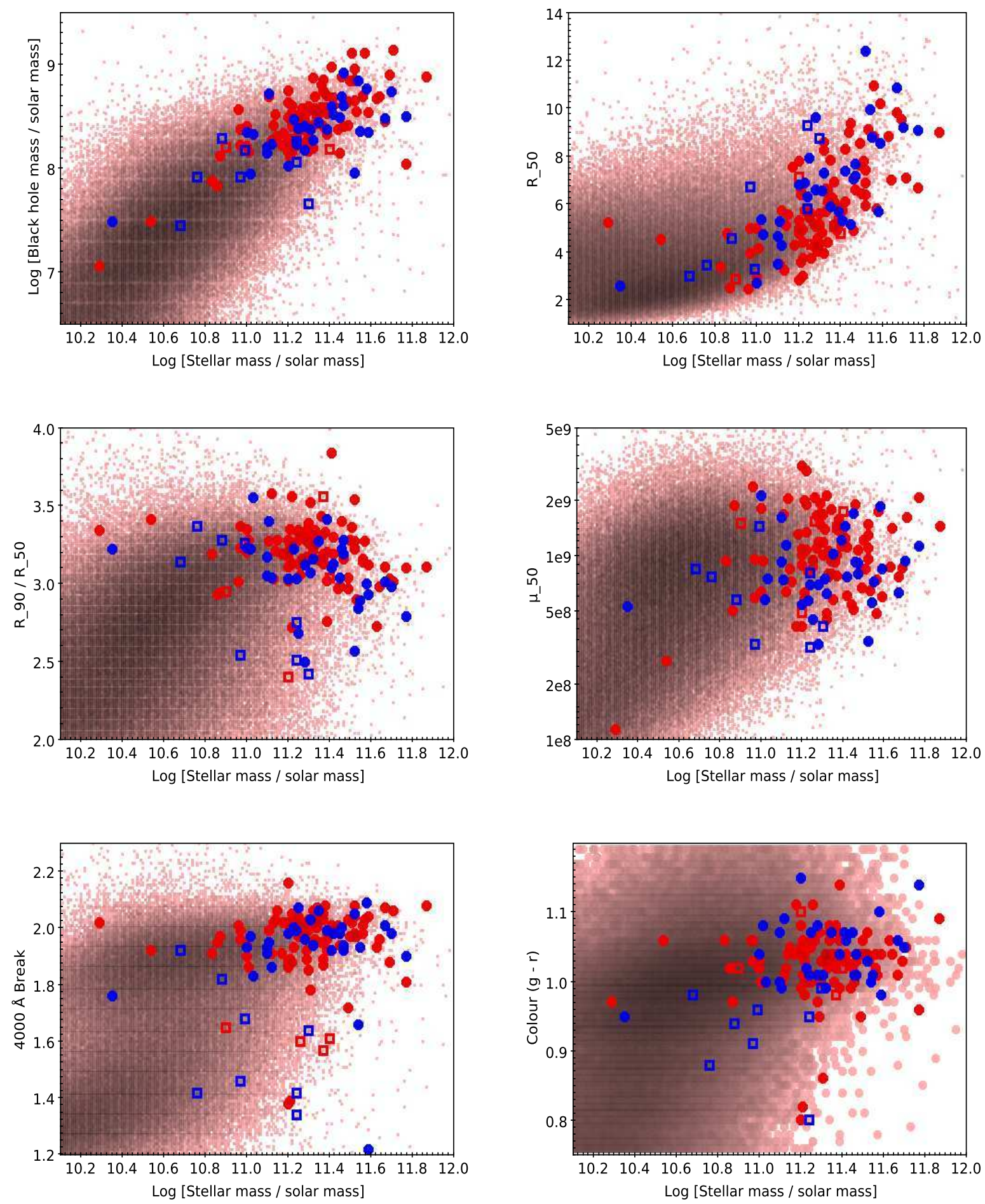

Figure 6. The host galaxy properties of the FRI (red) and FRII (blue) radio galaxies versus the stellar mass. The six plots show the black hole mass, galaxy half-light radius $\left(R_{50}\right)$, concentration index $\left(R_{90} / R_{50}\right)$, the stellar mass surface density $\left(\mu_{50}\right)$, the $4000 \AA$ break strength and the $g-r$ colour. The filled circles represent LERGs and open squares are HERGs. Pink circles are all galaxies.

and understand their causes to explain the morphological dichotomy observed at radio galaxies, we need to remove possible HERG/LERG biases. We also need to remove biases with the host galaxy mass and radio luminosity, since Figs 5 . 8 make clear that many parameters correlate strongly with these properties. The method we adopt for that in the next section is to construct populations of FRI vs FRII, HERG vs LERG and Compact vs Extended sources, having the same distribution of stellar mass, total radio luminosity or core radio luminosity, redshift and excitation class. We only confine the matching criteria to these parameters, in order to 

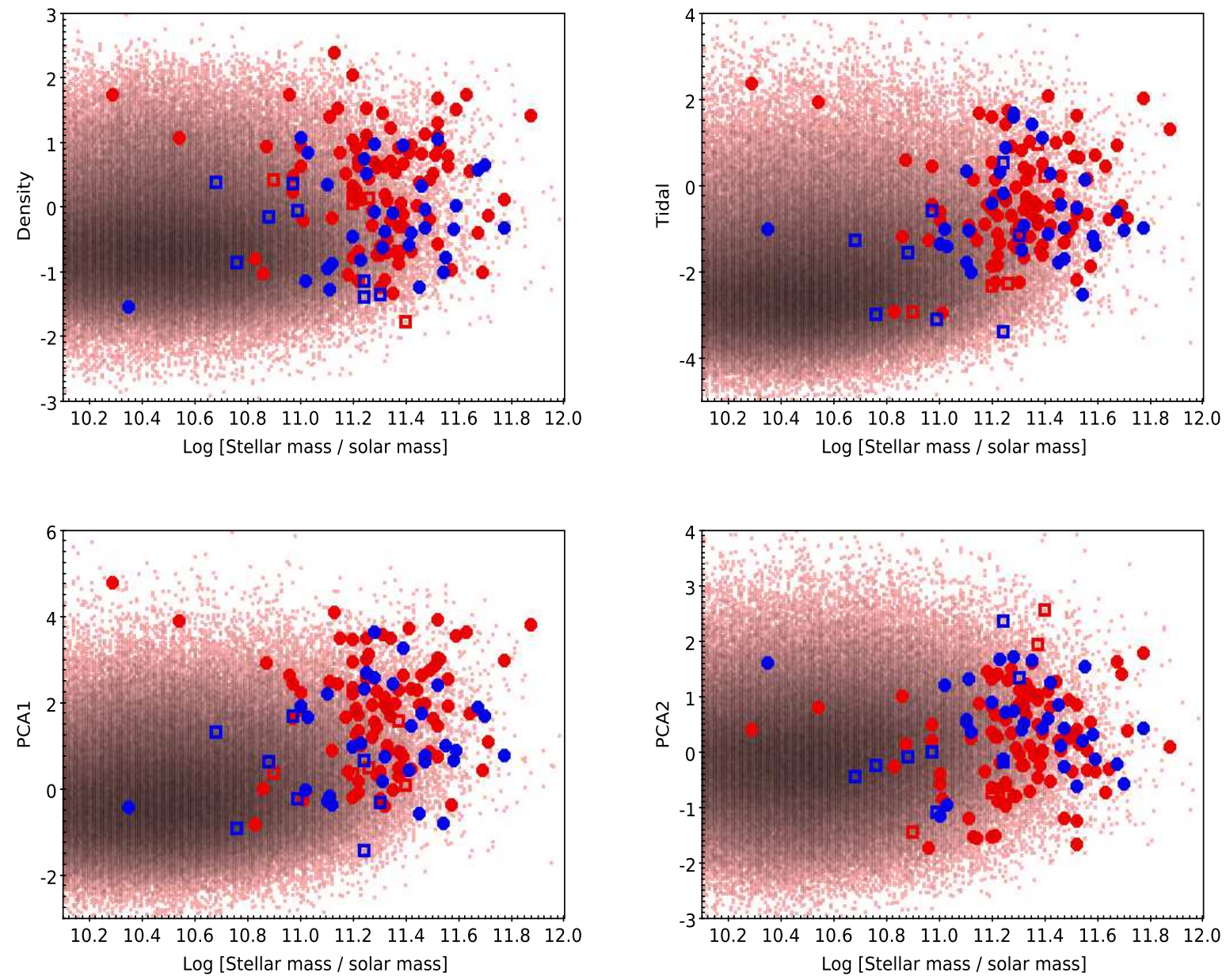

Figure 7. The environmental properties of the FRI (red) and FRII (blue) radio galaxies. The upper panels show the local galaxy density and the tidal interaction parameter plotted against stellar mass and the lower panels show two principal component parameters derived by Sabater et al (2013), each plotted against stellar mass. The filled circles represent LERGs and open squares are HERGs. Pink circles are all galaxies.
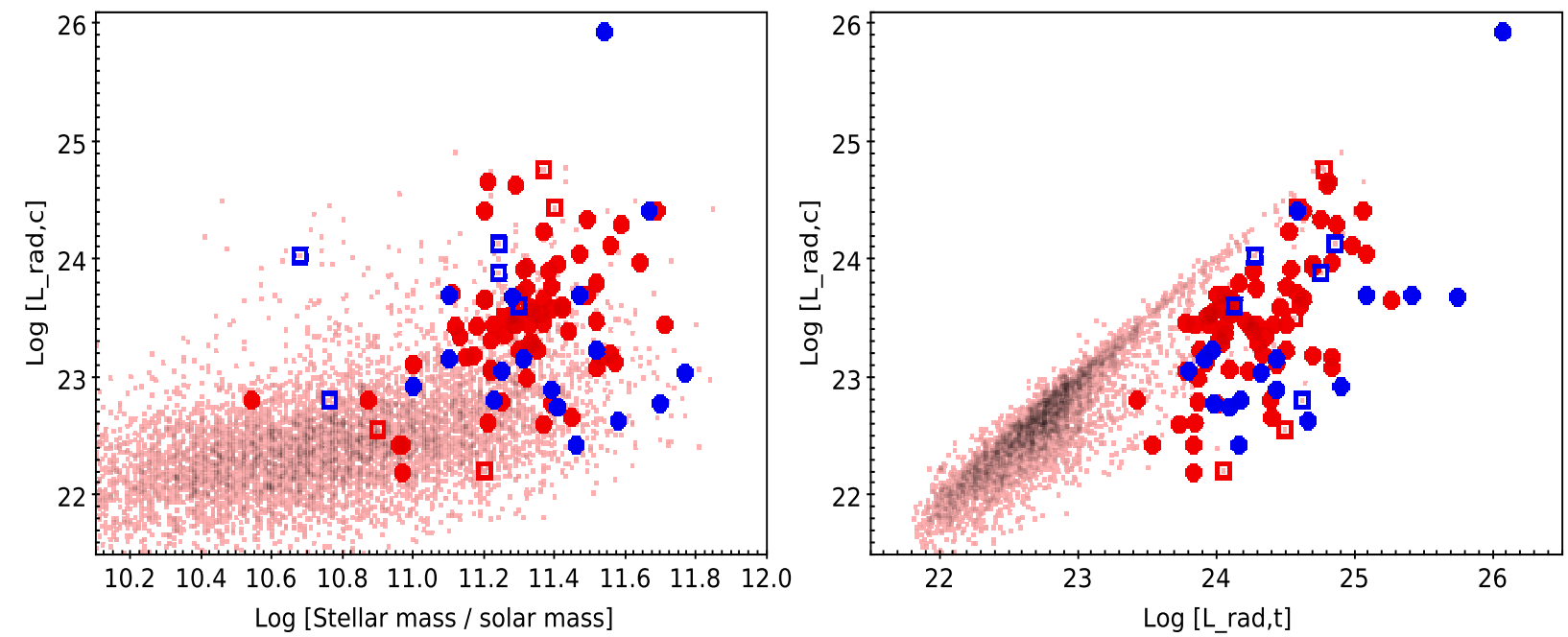

Figure 8. The core radio luminosity of the FRI (red) and FRII (blue) radio galaxies versus stellar mass (left panel) and total radio luminosity (right panel). The filled circles represent LERGs and open squares are HERGs. Pink circles are all galaxies. 
keep the sample of each type large enough to achieve robust statistics.

\section{MATCHED SAMPLES}

For each of FRI/FRII, HERG/LERG and Compact/Extended sources, we construct matched sample of objects in the $\mathrm{L}_{\text {radio- }}$ $\mathrm{M}_{\star}$ plane by randomly selecting pairs within a certain tolerance (detailed below) in $\mathrm{L}_{\text {radio }}$ and $\mathrm{M}_{\star}$ (two dimensional (2D) matching). Hence, we remove all of the mass-dependent and luminositydependent effects seen in the set of plots discussed in Section 3. We then consider the normalized cumulative histogram of each physical parameter for each class of objects, and apply the KolmogorovSmirnov (KS) test to assess whether the matched samples are consistent with being drawn from the same parent population. The KS test calculates the significance of the maximum difference (D) between two distributions, and assigns a probability $(\mathrm{P})$ according to the parameter $\mathrm{D}$ and the number of objects in the samples. We consider differences with a probability above 95 percent to be significant. Finally, we repeat the analysis up to 1000 times (with different random selections for the source pairing) and calculate the average $\mathrm{D}$ and then the significance from that.

We also constructed three dimensional (3D) matched samples by adding redshift $(\mathrm{z})$ to $\mathrm{L}_{\text {radio }}$ and $\mathrm{M}_{\star}$, and repeat the previous steps using a matched sample in $\mathrm{L}_{\text {radio }}-\mathrm{M}_{\star}-\mathrm{Z}$ space. In this way, we remove any effect of cosmic evolution (expected to be small) and more importantly any potential redshift biases in parameter estimation, in addition to the mass and luminosity. This results in smaller samples, due to the more restrictive matching requirements. The 3D-matching results are in good agreement with the 2D-matching results (but with larger uncertainties), so we only report 2D results in this section.

\subsection{FRI vs. FRII}

Here, we confine the analysis to FRI LERGs versus FRII LERGs. We want to remove all the effects caused by HERG/LERG nature of FR radio galaxies, and the LERG sample is numerous enough for both FR types to be well represented, while the HERG sample size is small (especially the FRI HERGs). As seen in the right-hand panel of Fig. [5 in a plane of $\mathrm{L}_{r a d, t}-\mathrm{M}_{\star}$, there is a slight segregation between FRIs and FRIIs, showing FRIIs have higher total radio luminosity on average (the median values of $\log \left[\mathrm{L}_{r a d, t}\right]$ for the FRIIs and FRIs are 24.44 and $24.29 \mathrm{~W} \mathrm{~Hz}^{-1}$ respectively). The matched sample has been constructed by finding all pairs within the error in radio luminosity $\Delta \log [\mathrm{L}]= \pm 0.2$ and in mass $\Delta \log [\mathrm{M}]=$ \pm 0.1 , and then choosing randomly unique pairs of FRI-FRII. The result of KS tests to investigate the significance of differences in the distribution of host galaxy and environment parameters between the two populations, averaged out of 1000 iterations, are presented in Table 5 The histograms for each of the parameters are presented in Fig. 9

There are a lot of differences with 99 percent significance presented in Table 5 FRIs have higher core radio luminosity than FRIIs with the same total radio luminosity, which emerges trivially from the definition of FRIs as being core dominated and edge darkened. Concerning the host galaxy properties, FRIs reside in smaller galaxies (lower $\mathrm{R}_{50}$ ) with higher concentration, higher mass surface density and higher $\mathrm{M}_{b h} / \mathrm{M}_{\star}$ (higher $\mathrm{M}_{b h}$ for the matched sample of mass), all of which imply less disk-like structure for the host galaxy. Concerning the environmental parameters, FRIs seem to lie in richer local environment: the density, PCA1 and richness are all higher for them than for FRIIs of the same mass, radio luminosity and excitation class, at high significance. The difference in tidal interaction is not significant. PCA2 shows the opposite behaviour, being higher in FRIIs.

These results suggest that extrinsic parameters can be the main driver of the morphological dichotomy. There are several indications for that. The first one is that FRIs have more concentrated host galaxies (higher $\left.\mathrm{R}_{90} / \mathrm{R}_{50}\right)$ with higher surface mass density $\left(\mu_{50}\right)$, indicating a greater density of material available to disrupt the radio jets. The second indication is that FRIs appear to reside in a denser galaxy environment, since all the environmental parameters tracing this seem to be higher for the FRIs compared to FRIIs. The only exception for that is the PCA2 parameter which is higher for FRIIs; this might show that FRIIs suffer a higher level of one-on-one interactions and are more likely to be merger/interaction triggered than FRIs (Miraghaei et al. 2014, 2015).

The picture that we can make from these results is that radio jets in denser galaxies, and in denser environments like galaxy clusters and groups, are much more susceptible to being disrupted and becoming FRI (cf. Kaiser \& Best 2007). These are also the environment in which giant elliptical galaxies have been formed, and so we observe less disk-like structures in them. The FRI galaxies may consequently be expected to be redder and less star forming, but these differences are not significant in our datasets, and might need a bigger sample size to be discovered. These results are consistent with the extrinsic scenario for the FR dichotomy. The one surprising result is observing higher [OIII] luminosity for FRI LERGs than matched FRII LERGs, with the high significance. This could be explained in the context of the extrinsic scenario, if there is more cold gas surrounding the nucleus, which converts the radiated luminosity more efficiently into line radiation but which is also capable of disrupting the radio jets. However, this could alternatively be due to higher levels of radiated luminosity from the core; this would not naturally fit into an extrinsic scenario, but could be interpreted as a selection effect caused by our matching in total radio luminosity, and FRIs being more core dominated sources, since the core radio luminosity seems to be better correlated with the [OIII] line emission in FRIs than total radio luminosity is (Baldi, Capetti \& Giovannini 2015; see also Section 4.3).

\subsection{HERG vs. LERG}

In order to compare HERGs and LERGs, we construct each sample by combining HERG and LERG sources from three different classes of FRI, FRII and compact radio AGN. We make the matched sample by cross-matching HERG objects with LERG objects from the same class, to remove the morphological effects caused by this method. Fig. 10 shows the radio luminosity versus stellar mass distribution of all FRI, FRII and compacts separated into HERGs and LERGs. There are relatively few HERG sources while LERGs are more populated in each class of FRI/II and compact. A one-to-one matching scheme thus result in a small sample of HERG/LERG and large uncertainties for the comparison. Therefore, we cross-match each HERG with three different LERGs, which is possible due to mismatch in HERG and LERG numbers, and helps to improve the overall sample size and significance. We also allow a wider matching tolerance for the differences in radio luminosity $(\Delta \log [\mathrm{L}]= \pm 0.5)$ and mass $(\Delta \log [\mathrm{M}]= \pm$ $0.2)$ that will help with the random selection of matches. Finally, as is clear on Fig. 10, there are five low mass FRII HERGs with only a few FRII LERGs around them, which are insufficient to match all 


\begin{tabular}{|c|c|c|c|c|c|c|c|c|}
\hline Sample & \multicolumn{2}{|c|}{ FRII-FRI } & \multicolumn{2}{|c|}{ HERG-LERG } & \multicolumn{4}{|c|}{ Compact-Extended } \\
\hline $\begin{array}{l}\text { Matched properties } \\
\text { Sample size }\end{array}$ & \multicolumn{2}{|c|}{$\begin{array}{c}\mathrm{L}_{r a d, t}-\mathrm{M}_{\star} \\
\mathrm{M}=\mathrm{N}=77\end{array}$} & \multicolumn{2}{|c|}{$\begin{array}{l}\mathrm{L}_{\text {rad, } t}-\mathrm{M}_{\star} \\
\mathrm{M}=15, \mathrm{~N}=45\end{array}$} & \multicolumn{2}{|c|}{$\begin{array}{c}\mathrm{L}_{r a d, t}-\mathrm{M}_{\star} \\
\mathrm{M}=\mathrm{N}=81\end{array}$} & \multicolumn{2}{|c|}{$\begin{array}{c}\mathrm{L}_{r a d, c}-\mathrm{M}_{\star} \\
\mathrm{M}=\mathrm{N}=58\end{array}$} \\
\hline Significance thresholds & $\mathrm{D}_{95}=\mathrm{C}$ & $99=0.26$ & $\mathrm{D}_{95}=\mathrm{C}$ & $99=0.48$ & $\mathrm{D}_{95}=$ & $9=0.26$ & & $9=0.30$ \\
\hline $\mathrm{L}_{r a d, c}$ & 0.58 & $>99 \%$ & -0.36 & - & -0.68 & $>99 \%$ & - & - \\
\hline $\mathrm{L}_{\text {rad,t }}$ & 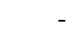 & - & 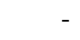 & - & - & - & 0.57 & $>99 \%$ \\
\hline $\mathrm{R}_{50}$ & -0.35 & $>99 \%$ & -0.10 & - & 0.17 & - & -0.05 & - \\
\hline g-r & 0.21 & - & 0.36 & - & 0.18 & - & -0.07 & - \\
\hline $4000 \AA$ break & 0.20 & - & 0.74 & $>99 \%$ & 0.33 & $>99 \%$ & 0.06 & - \\
\hline $\mathrm{R}_{90} / \mathrm{R}_{50}$ & 0.32 & $>99 \%$ & 0.45 & $>95 \%$ & 0.17 & - & 0.20 & - \\
\hline$\mu_{50}$ & 0.38 & $>99 \%$ & -0.30 & - & -0.20 & - & 0.22 & - \\
\hline $\mathbf{M}_{B H}$ & 0.35 & $>99 \%$ & 0.47 & $>95 \%$ & 0.16 & - & 0.35 & $>99 \%$ \\
\hline Density & 0.36 & $>99 \%$ & 0.43 & $>95 \%$ & 0.12 & - & 0.09 & 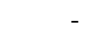 \\
\hline Tidal & 0.15 & - & 0.42 & $>95 \%$ & 0.18 & - & 0.14 & 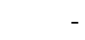 \\
\hline Richness & 0.28 & $>99 \%$ & 0.24 & - & 0.13 & - & 0.14 & - \\
\hline PCA1 & 0.30 & $>99 \%$ & 0.53 & $>99 \%$ & 0.13 & - & 0.09 & - \\
\hline PCA2 & -0.31 & $>99 \%$ & -0.19 & - & 0.19 & - & 0.07 & - \\
\hline $\mathrm{L}_{[O I I I]}$ & 0.38 & $>99 \%$ & -0.90 & $>99 \%$ & -0.30 & $>99 \%$ & -0.13 & - \\
\hline
\end{tabular}

Table 5. The result of KS test for three sets of comparisons: i) FRI and FRII radio galaxies ii) HERGs and LERG sources iii) compact and extended source. They have been cross-matched in luminosity-mass plane where $\mathrm{L}_{r a d, t}$ represents total radio luminosity and $\mathrm{L}_{r a d, c}$ represents core radio luminosity. The first column in each set shows the KS differences D, and the second column shows confidence level for the estimated differences. We only indicate the significance above 95 percent. Positive values show that the first mentioned sample in each set (Compact, FRII and HERG) has lower value for the declared characteristic and negative sign means that the first mentioned sample has higher value. For example, FRIIs have higher $\mathrm{R}_{50}$ compared to FRIs with $>99$ percent confidence and HERGs have lower black hole mass with $>95$ percent confidence. The typical uncertainty of the D values (the standard deviation out of 1000 iterations) is 0.01-0.03 which we have considered to report the probabilities. $\mathrm{M}$ and $\mathrm{N}$ are the sizes of the first and the second mentioned sample in each set which we have used to calculate significance thresholds (D). $\mathrm{D}_{95}$ and $\mathrm{D}_{99}$ are the level of D needed for $95 \%$ and $99 \%$ significance respectively.
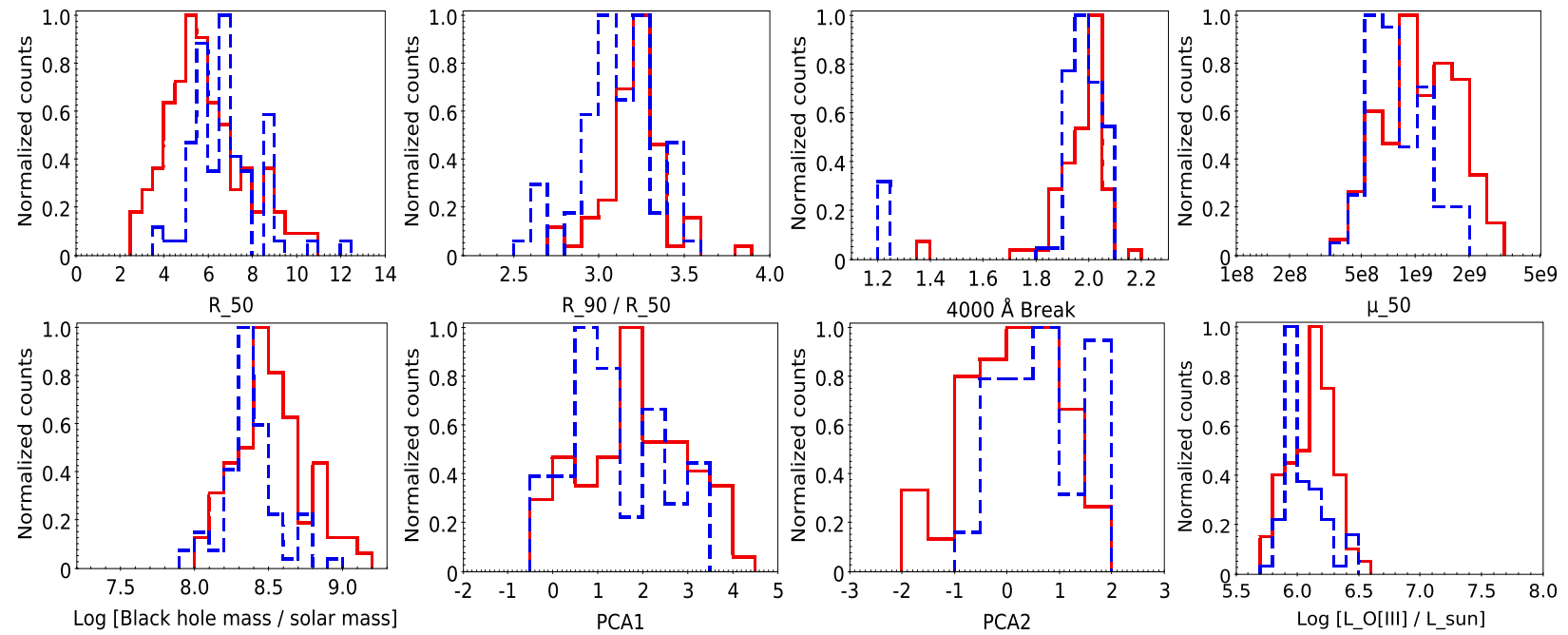

Figure 9. Histograms of the host galaxy and environmental parameter distributions for FRI (red) and FRII (blue) radio galaxies matched in LERG classification and in the $\mathrm{L}_{r a d, t}-\mathrm{M}_{\star}$ plane.

5 HERGs. Thus, in each iteration we randomly choose two HERGs and cross-matched them with the three FRII LERGs each, within a wider range of radio luminosity $(\Delta \log [\mathrm{L}]= \pm 1.0)$ and mass $(\Delta \log [\mathrm{M}]= \pm 0.4)$ differences. By these methods, we have constructed significant-sized samples of HERGs (15) and LERGs (45) with the same distribution of stellar mass, total radio luminosity and morphology.

The results of the comparison of host galaxy and environmental properties, confirmed by KS test, are presented in Table 5 and the histograms for each of the parameters are presented in Fig. 11 Differences with over 95 percent confidence have been detected for both environmental and host galaxy parameters. In terms of host galaxy properties, HERGs are younger with lower concentration and lower black hole mass (thus, lower $\mathrm{M}_{b h} / \mathrm{M}_{\star}$ ) than LERGs, indicating that they reside in more disky galaxies, as previously reported by Best \& Heckman (2012). The significance in our study is lower for some correlations than was found by Best \& Heckman, because of the smaller sample size, but importantly we have eliminated any possible biases associated with FRI/II classifications. Therefore, our results are robust. HERGs also have higher [OIII] luminosity, as expected from their definition as sources with a stronger ionising component. The environments of HERGs appear to show lower density and tidal interactions than those of LERGs. The significance of the PCA1 parameter analysis confirms the lower density environment for HERGs, while the lack of any difference in PCA2 distributions shows that the apparently lower 


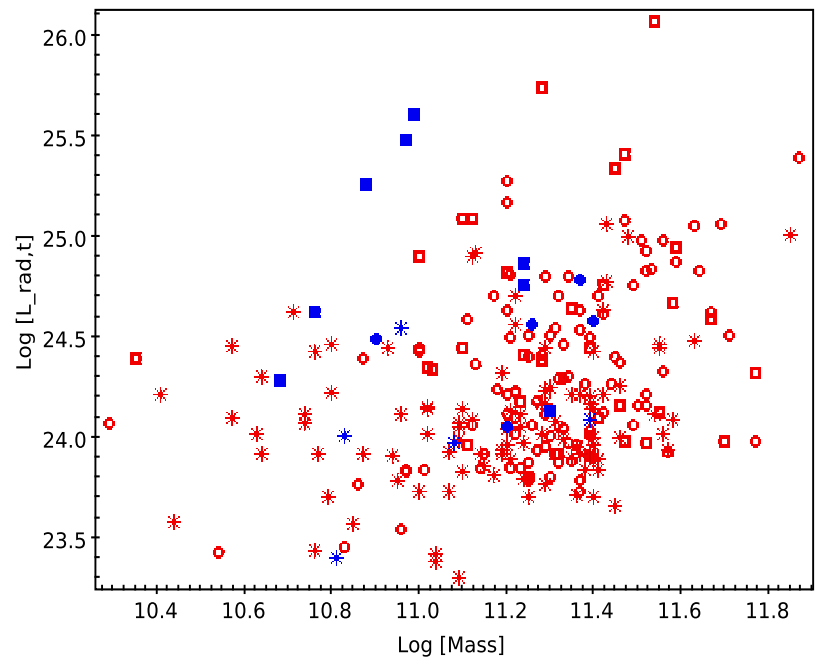

Figure 10. Total radio luminosity versus stellar mass of FRIs (open and filled circles), FRIIs (open and filled squares) and compact radio AGN (stars). The colour red represents LERGs and blue represents HERGs.

tidal interaction in HERGs might be a projection effect. These environmental results are consistent with those of Gendre et al. (2013) who have reported low density environments for HERGs independent of FR morphology.

It is worth mentioning that by comparing HERGs and LERGs using a sample of FRII HERGs and FRII LERGs, without crossmatching for luminosity and mass, we get the same result but with lower significance.

These results are consistent with the currently favoured description of HERG and LERG origins, which associated the differences to Eddington-scaled accretion rates on to the black hole (see discussions in Heckman \& Best 2014). HERGs require high accretion rates fuelled from extensive cold gas reservoirs; this gas-rich environment is more readily available in later-type disky galaxies with lower concentration and higher star formation, as seen in the data. Their low density environments are also consistent with their fueling mechanisms, in a sense that in high density environments galaxies tend to be gas-poor, due to a combination of processes including stripping and strangulation (cf. Boselli \& Gavazzi 2006). In contrast, galaxy groups and clusters have giant elliptical galaxies in their center with the high black hole masses, high concentration, old stellar population and little cold gas remaining to feed the central nuclei, but do have the cooling of hot gas which can provide the low accretion rates necessary to fuel LERGs (e.g. Best \& Heckman 2012): these are exactly the properties found for the LERG sources.

These arguments can also help to explain the overlap of FRIIs with HERGs and FRIs with LERGs; these have their origin in both radio luminosity and environment. The higher accretion rates required to fuel HERGs also lead to more powerful radio jets, which are more likely to be able to survive the disrupting effects of their surrounding environments and become FRII sources; only as minority form FRIs. In contrast, at the lower accretion rates of LERGs, the lower power jets are more likely to be disrupted and become FRIs, although there remains a significant population of LERG FRIIs where the jets manage to survive. This connects to the host galaxy and surrounding environment, responsible for disrupting the jets, which also provides links between the FR classification and the excitation state. FRIIs and HERGs both are favoured in lower density environments and later-type galaxies, since these both offer a more plentiful supply of cold gas to provide higher fu- elling rates, and less potential to disrupt the jets. FRIs and LERGs are developed in early type galaxies and higher density environment, in both of which the gas supplies are likely to be limited, and jets more easily disrupted.

\subsection{Extended vs. Compact}

In this section, we investigate compact radio AGN and compare them with the extended FR radio sources. The main question that we address is which scenarios for the origin of compact AGN fit best with the observations: are the compact sources a fundamentally different class of objects, are they FR radio galaxies at the early stage of their evolutions, or are they short-lived sources which die before they extend to large distances?

The selection criteria for each class are presented in Section 2. Fig. 12 shows the total and core radio luminosity of both the compact and extended samples versus stellar mass. Compact sources have, on average, lower total radio luminosity while extended radio sources have lower core radio luminosity. Accordingly, we set up two different comparisons, creating a sample matched in total radio luminosity and stellar mass, and a second sample matched in core radio luminosity and stellar mass. Both core radio luminosity and total radio luminosity have been used to estimate the jet power (Kording, Jester \& Fender 2008). While there is a tight correlation between these two parameters in low luminosity sources (with little extended emission), the correlation shows a very large scatter for the very luminous radio sources which are subject of this work (see Fig. 8, right panel). The core luminosity has been argued to be a better gauge of jet power than total radio luminosity, as it is a measure of instantaneous power, rather than something averaged over time and influenced by environment; even at fixed jet power, the total luminosity of a radio source evolves as the source grows, going first up then down according to current models of radio source growth (e.g. Kaiser \& Alexander 1999; Turner \& Shabala 2015). On the other hand, core luminosity may sometimes be affected by relativistic beaming (see the discussion in, e.g., Marcha et al. 2005; Sadler et al. 2014). Total luminosity might be a good gauge if the compact sources were simply small, caught early in their life and perhaps shorter lived than FRI/II. Considering both core and total radio luminosity in this section will help to identify whether the results obtained by the two methods match or differ, thus giving an idea which is the better comparison.

In order to make matched samples we adopted the same method that we used in Section 4.1. Here, we make the matched samples in both the $\mathrm{L}_{r a d, t}-\mathrm{M}_{\star}$ and $\mathrm{L}_{\text {rad,c }}-\mathrm{M}_{\star}$ planes with matching-tolerance limits of $\Delta \log [\mathrm{L}]= \pm 0.2$ and $\Delta \log [\mathrm{M}]=$ \pm 0.1 . The KS-test results for the comparison of host galaxy and environment parameters are listed in Table 5, and histograms for each of the parameters are presented in Figs. 13 and 14

If we assume that total radio luminosity is a good measure for the average jet power, then this match in total radio luminosity will select a sample of compact and extended objects matched in jet power, and then we can investigate which characteristics drive the compact-extended dichotomy. Compact and extended objects with the same distribution of total radio luminosity show $>95 \%$ significant differences in only $4000 \AA$ break and [OIII] luminosity: compact objects have younger stellar populations and higher line luminosity, both of which imply there is more cold gas available either for star formation or AGN fueling in these objects. There is also a consistent trend of differences (but below 95 percent significance in each individual case) in concentration, colour and size of the host galaxy, all of which point towards compact radio sources 

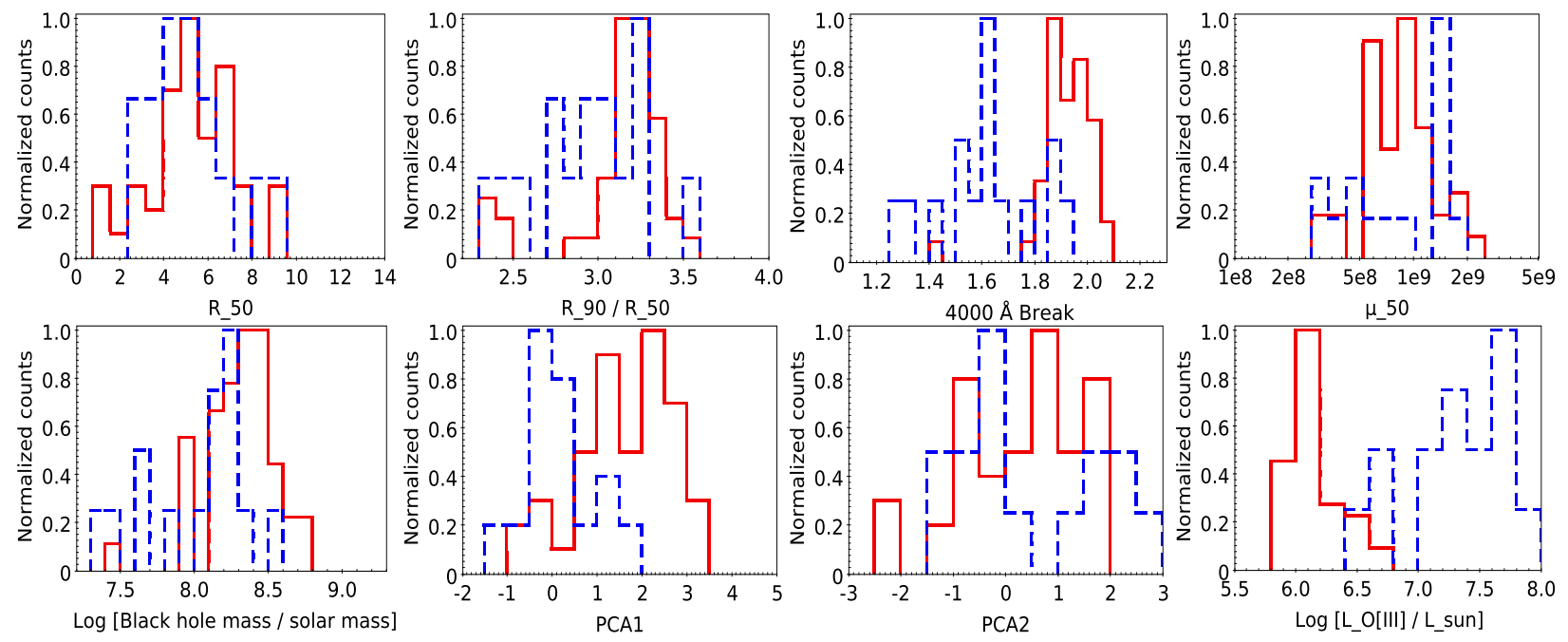

Figure 11. Histogram of the host galaxy and environmental parameter distributions for LERG (red) and HERG (blue) radio sources, matching in radio morphology and in the $\mathrm{L}_{r a d, t}-\mathrm{M}_{\star}$ plane.
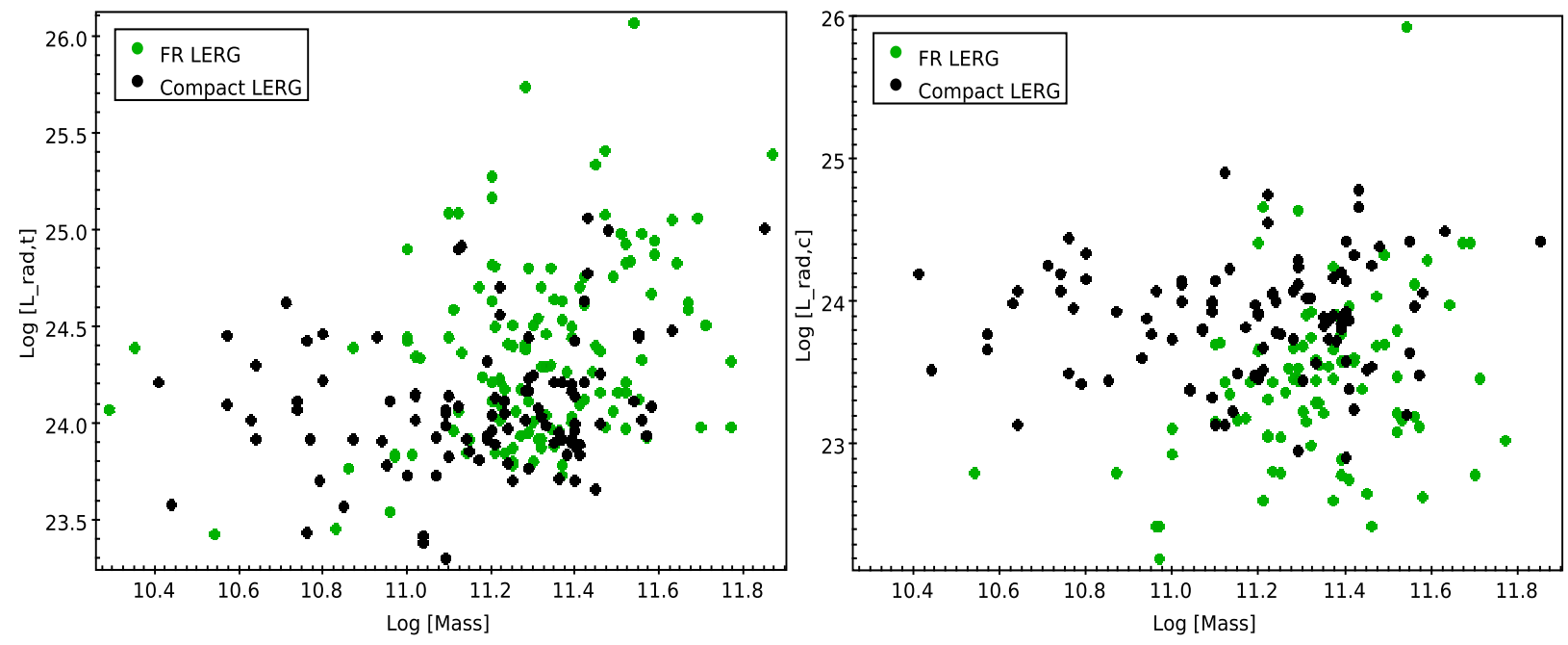

Figure 12. Total radio luminosity (left panel) and core radio luminosity (right panel) versus the stellar mass of the extended radio galaxies (green) and compact radio galaxies (black).

being found in galaxies with stronger disk-like components, consistent with the higher star-formation rates. No significant environmental differences have been detected. Prestage \& Peacock (1988) previously argued that compact radio sources lie in regions of lower galactic density than extended sources; our results show a weak trend in that direction, but at below $95 \%$ statistical significance. It is possible that their result was partially driven by stellar mass and/or radio luminosity differences between their samples. Finally, the results show that the matched samples of compact and extended sources have similar distribution of black hole mass, which supports the correlation of black hole mass and average jet power.

Interesting results come out when we matched the radio luminosity of the core for both samples. In this case, the only significant difference that is observed between the two samples is in the black hole mass. There is no difference in [OIII] luminosity. This result shows that core radio luminosity is correlated to the [OIII] luminosity, as previously discussed by Baldi, Capetti \& Giovannini (2015). They showed that compact LERGs lie in the same region of the [OIII] versus core luminosity plane that FRI LERGs do. There- fore, when we matched in core radio luminosity we consequently matched in [OIII] luminosity. The second important result is that again no environmental differences have been detected. This completely rules out a scenario in which jet disruption by the dense galactic and intergalactic environment causes the radio morphological differences between compact and extended objects. It also rules out any model in which equivalent jets are launched in the two cases, but that in low density environments the lack of a strong working surface causes the jet to escape without producing luminous extended radio emission (giving the impression of a compact source).

The most important result is that, by having the same environmental and host galaxy properties observed in core-matched compact and extended sources, the morphological differences appear to have their origin in the black hole mass. The lower black hole mass in compact sources seems to be less efficient at launching stable large-scale radio jets, or is able to support these jets for much shorter periods of time. This result is consistent with these of Baldi, Capetti \& Giovannini (2016) who claim compact sources (or type 

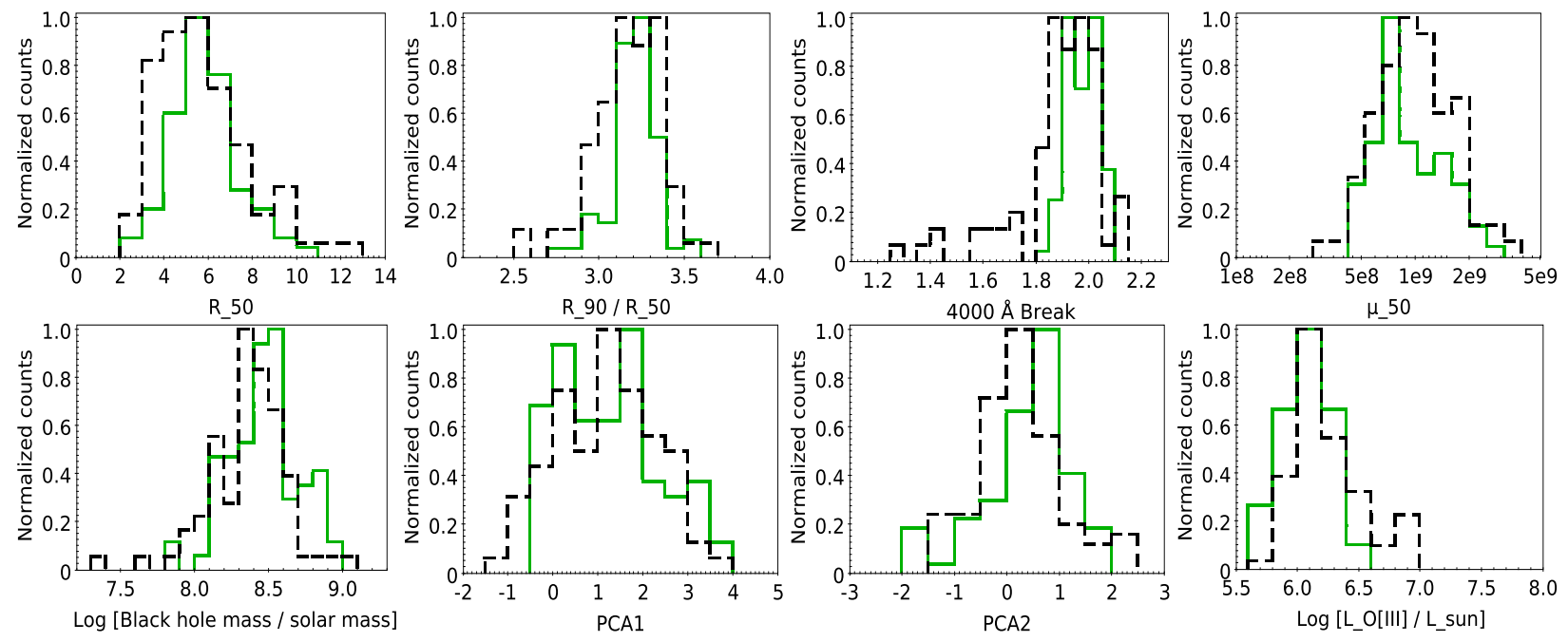

Figure 13. Histogram of the host galaxy and environmental parameter distributions for compact (black) and extended (green) radio sources matched in the $\mathrm{L}_{\text {rad,t }}-\mathrm{M}_{\star}$ plane.
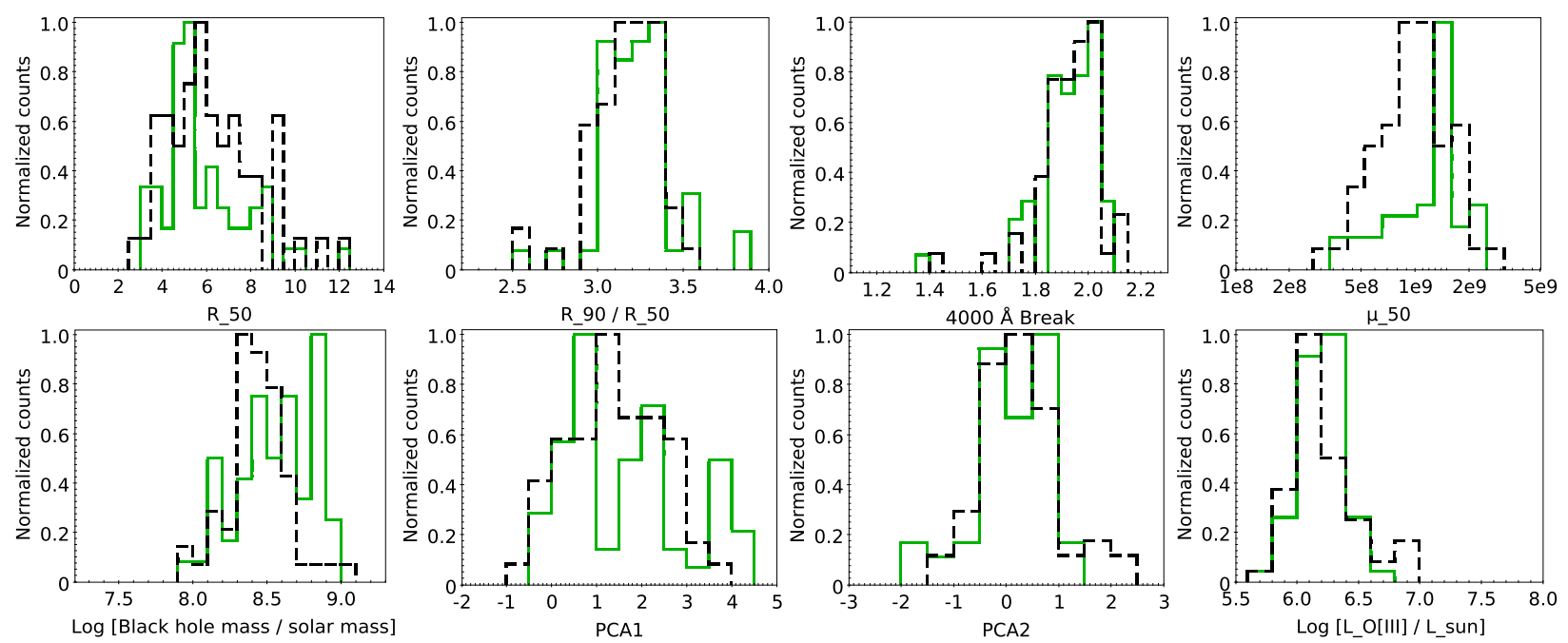

Figure 14. Histogram of the host galaxy and environmental parameter distributions for compact (black) and extended (green) radio sources matched in the $\mathrm{L}_{\text {rad,c }}-\mathrm{M}_{\star}$ plane.

FR0s, as they named compact LERGs) have smaller jet Lorentz factor compared to FRIs. On a broader scale, a black-hole mass $v s$ jet launching efficiency correlation would also explain the very strong correlation seen between black hole mass and the fraction of galaxies that host radio-loud AGN (e.g. Best et al. 2005b).

Generally, the robust conclusions out of these two comparisons are that compact objects can not simply be FR radio galaxies at the early stage of their evolutions, or viewed at small angle to their axis, as these models could not account for the observed differences in the host galaxy parameters of the compact and extended sources in our samples. (More specifically, some of the compact objects may well be caused by one of these effects, but the full population cannot be - there must also be other effects at work). Furthermore, the differences are not driven in any way by different environments of the sources. Rather, there must be a fundamental difference between the objects, with the compact objects either being short-lived radio sources disrupted before they expand to large scale, or objects which do not efficiently launch large-scale radio jets, perhaps due to their lower mass black holes.

\section{BEYOND THE NORMAL FR RADIO GALAXIES}

As we have described in Secton 2.1, there are several extended sources with different and more complex morphologies compared to the normal class of FRI/II radio galaxies; these are flagged as double-double (D-D) sources (Schoenmakers et al. 2000), head-tail (HT) sources (Rudnick \& Owen 1976), wide-angle tailed (WAT) sources (Owen \& Rudnick 1976) and FR hybrid (FRH) sources (Gopal-Krishna \& Wiita 2000). In this section, we focus on these types of radio sources and explore the host galaxy and the environment properties of them to see what light this may shed on what causes such complex morphologies. For this purpose, we applied the redshift cuts described in Section 2, which greatly decreases the number of sources but provides an unbaised framework to look at these sources among normal FRI/II radio galaxies. Fig.15 shows the results. Some examples of these sources in our sample are shown in Fig. 1

Given the small sample sizes, only qualitative conclusions can be drawn. The WATs and HT lie systematically towards the lower 

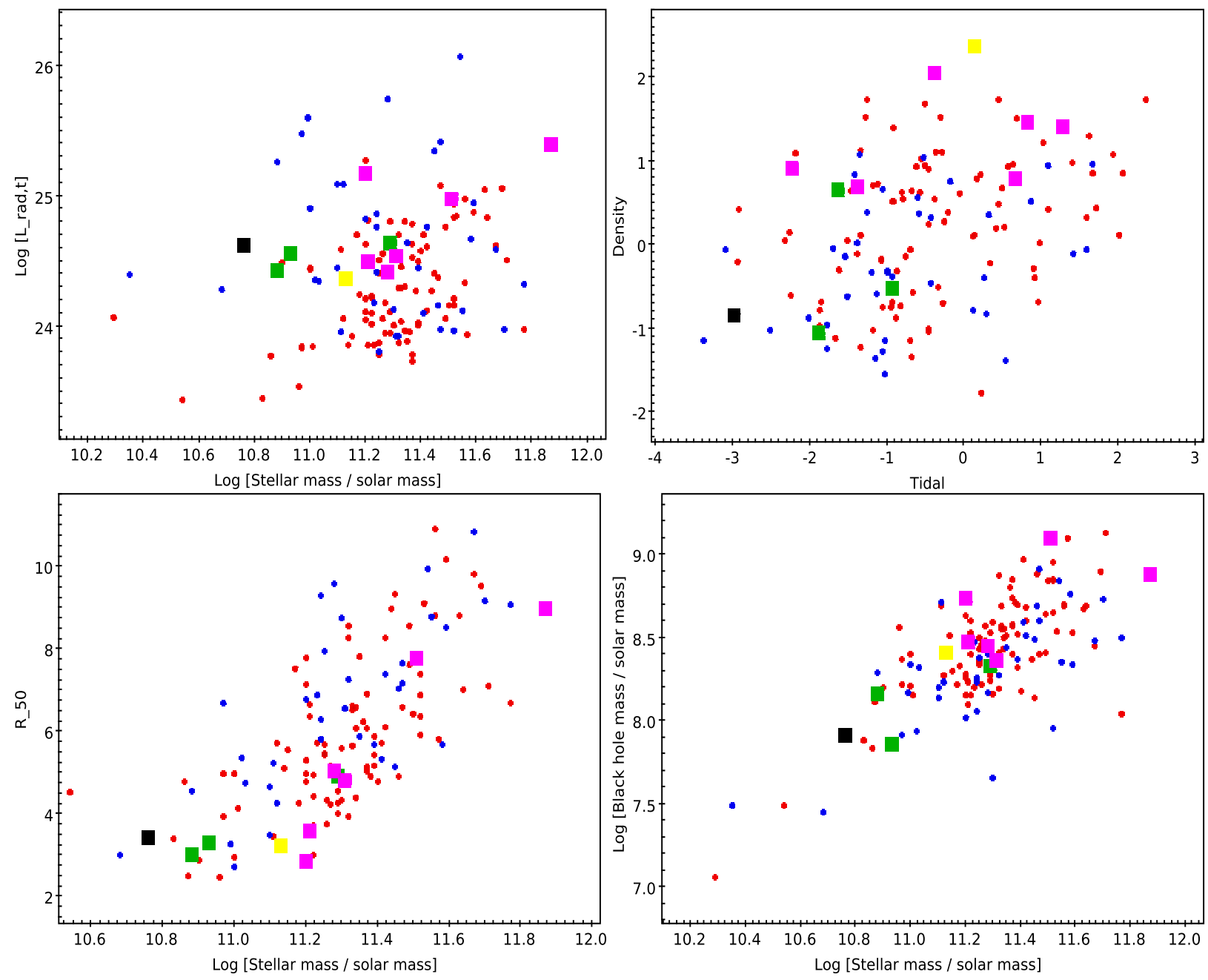

Figure 15. The host galaxy and environment properties of FRI (red circle), FRII (blue circle), HT (yellow square), WAT (pink square), hybrid (green square) and D-D (black square) radio galaxies.

part of the $R_{50}-M_{\star}$ distribution, suggesting that they have smaller host galaxies compared to FRIs and FRIIs. They also seem to have higher total radio luminosity. The clearest result, however, comes from the environment properties of these sources. HT and WATs are found to reside in the densest environments, which is exactly as expected since these are understood to be shaped when the radio jet emission is bent by the relative movement of the galaxies through the intra-cluster medium. Therefore, WAT and HT can be efficiently used to identify overdensities (Blanton et al. 2000, 2001; Dehghan et al. 2014; O'Brien et al. 2016) especially in the distant universe where the current resolution and sensitivity of X-ray observations do not allow deep exploration. This study provides a rich sample of WAT (53) and HT (9) radio sources (see Table 1), distributed over the redshift range of 0.03-0.4, and deeper radio surveys will soon allow these to be selected to higher redshifts.

Likewise, although there is only one D-D source, it is interesting that this lies exactly at the lowest density part of the density-tidal plane. Again this is what would be expected, since D-D sources are usually giant radio galaxies, and low density environments allow these to be achieved by the jet expanding freely. The FRHs are not found in any special region of parameter space, although we can't make any strong statements on the basis of just a few sources. More information about these FRHs could be gleaned by deriving and examining host galaxy and environment parameters for the wider sample of 35 sources presented over the full redshift range in Table 1 but this is beyond the scope of the current paper.

\section{SUMMARY AND CONCLUSIONS}

We have studied powerful radio galaxies with a wide range of radio structures, from compact to very extended double-lobe radio sources, and with a very different optical spectrum, in order to understand the origin of the observed differences. The radio sources and their corresponding host galaxies were obtained from Best \& Heckman (2012) who have cross-matched DR7 of the SDSS with the NVSS and FIRST catalogues. The radio galaxy sample has been divided into compact and extended radio sources according to their radio morphologies. The extended radio galaxies known as Fanaroff-Riley (FR) radio galaxies have been visually divided into type I (FRI) and type II (FRII), with a few additional sources classified as hybrid, wide-angle tailed, head-tail and double-double radio galaxies. The resultant catalogue, which is presented here, provides a precious sample of over a thousand FR-classified radio galaxies brighter than $S_{1.4 \mathrm{GHz}}=40 \mathrm{mJy}$, out to $z \approx 0.4$.

The subset of radio sources with $0.03<z<0.1$ have 
also been divided into high excitation (HERG) and low excitation (LERG) radio galaxies according to their optical spectrum. HERG and LERG sources are understood to correspond to the sources with high rate of cold accretion flow and low rate of hot accretion flow respectively. The purpose of this paper was to investigate the differences in the host galaxies and environment of the FRI/II and compact sources with HERG and LERG nature separately, in order to disentangle which effects cause each of the FRI/II, compact/extended and HERG/LERG dichotomies.

We investigated the FRI/FRII dichotomy using a sample of FRI LERGs and FRII LERGs with the same stellar mass and total radio luminosity distribution, to remove any biases caused by HERG/LERG nature, mass and radio luminosity. We show that FRIs are hosted by smaller galaxies with higher concentration, higher mass surface density, and higher black hole to stellar mass ratio than the FRIIs, consistent with the galaxies possessing less disky structure. The environment of the FRI radio galaxies show higher density and richness. All the results are consistent with the models that employ extrinsic parameters (i.e. jet disruption by the interstellar and intergalactic media) to explain the FRI/FRII dichotomy. Previous studies that focussed on intrinsic differences were all biased by the HERG/LERG classification in the sense that most of them compare FRI LERGs with the FRII HERGs.

We investigated the environment and the host galaxy properties of HERGs and LERGs using a sample of combined FRI/II and compact HERGs with FRI/II and compact LERGs, matched in classification, mass and total radio luminosity. We confirm that HERGs are hosted by galaxies with smaller $4000 \AA$ break, higher [OIII] luminosity and lower black holes mass with bluer colour and lower concentration compared to the LERGs - independent of FR classification. These all indicate that HERGs are found in more star-forming and disky galaxies. The environments of LERGs display higher density compared to the HERGs. These results support the hypothesis that the AGN fueling source is the main origin of HERG/LERG dichotomy. In dense environments and massive elliptical galaxies the AGN fueling source is believed to be primarily the hot intergalactic gas which cools and accretes on to the central black hole at a low accretion rate, giving rise to LERGs. In low density environments (without hot haloes), depending on the availability of cold gas, HERG radio sources may form. Therefore, HERG sources are found in more star-forming and disky galaxies, more typically in lower density environments, with a higher prevalance of cold gas.

We also investigated the compact/extended dichotomy by comparing a combined sample of FRI and II LERGs with a sample of compact LERGs, matched in stellar mass and either core or total radio luminosity. In neither case did we find any difference in the AGN environment, indicating that this is not a cause of the dichotomy. We confirm that the [OIII] luminosity distributions are the same when matched in core radio luminosity but not in total radio luminosity, suggesting that the core radio luminosity is the better measure of the current accretion power. In the core luminosity matched samples, the only parameter which showed a significant difference between compact and extended radio sources is the black hole mass: compact objects harbour lower mass black holes. This result implies that lower mass black holes are either less efficient at launching stable large-scale radio jets (consistent with the interpretation of Baldi et al. 2016), or able to do so for a shorter time such that these sources are short-lived.

Finally, we explored the host galaxy and environment properties of radio galaxies with more complex and interesting morphologies such as wide-angle tailed, head-tail, double-double, and
FR hybrid. Although the samples are too small to draw quantitative conclusions, we confirm that HT and WAT reside in very dense regions compared to the whole population, offering the prospect to identify over-dense regions such as galaxy clusters and groups through radio observation alone. This will be a powerful tool in next-generation radio surveys.

\section{ACKNOWLEDGMENTS}

H. M. would like to thank Institute for Astronomy Royal Observatory Edinburgh for partial financial support. PNB is grateful for support from the UK STFC via grant ST/M001229/1.

\section{REFERENCES}

Abazajian K. N., Adelman-McCarthy J. K., Agueros M. A., et al. 2009, ApJS, 182, 543

Alexander P., 2000, MNRAS, 319, 8

Baldi R. D., Capetti A., Giovannini G., 2015, A\&A, 576, 11

Baldi R. D., Capetti A., Giovannini G., 2016, AN, 337, 114

Baum S. A., Heckman T. M., van Breugel W., 1992, ApJ, 389, 208

Baum S. A., Zirbel E. L., O’Dea C. P., 1995, ApJ, 451, 88

Becker R. H., White R. L., Helfand, D. J., 1995, ApJ, 450, 559

Best P. N., Kauffmann G., Heckman T. M., Ivezic, Z., 2005, MNRAS, 362, 9

Best P. N., Kauffmann G., Heckman T. M., Brinchmann J., Charlot S., Ivezic Z., White S. D. M., 2005, MNRAS, 362, 25

Best P. N., von der Linden A., Kauffmann G., Heckman T. M., Kaiser C. R., 2007, MNRAS, 379, 894

Best P. N., 2009, AN, 330, 184

Best P. N., Heckman T. M., 2012, MNRAS, 421, 1569

Best P. N., Ker L. M., Simpson C., Rigby E. E., Sabater J., 2014, MNRAS, 445, 955

Blandford R. D., Konigl A., 1979, ApJ, 232, 34

Blanton E. L., Gregg M. D., Helfand D. J., Becker R. H., White R. L., 2000, ApJ, 531, 118

Blanton E. L., Gregg M. D., Helfand D. J., Becker R. H., Leighly K. M., 2001, AJ, 121, 2915

Boselli A., Gavazzi G., 2006, PASP, 118, 842, 517

Brinchmann J., Charlot S., White S. D. M., Tremonti C., Kauffmann G., Heckman T., Brinkmann J., 2004, MNRAS, 351, 1151

Buttiglione S., Capetti A., Celotti A., Axon D. J., Chiaberge M., Macchetto F. D., Sparks W. B., 2010, A\&A, 509, 15

Ceglowski M., Gawronski M. P., Kunert-Bajraszewska M., 2013, A\&A, 557, 6

Cid Fernandes R., Stasinska G., Schlickmann M. S., Mateus A., Vale Asari N., Schoenell W., Sodre L., 2010, MNRAS, 403, 1036 Clewley L., Jarvis M. J., 2004, MNRAS, 352, 909

Condon J. J., Cotton W. D., Greisen E. W., Yin Q. F., Perley R. A., Taylor G. B., Broderick J. J., AJ, 115, 1693

Dehghan S., Johnston-Hollitt M., Franzen T. M. O., Norris R. P., Miller N. A., 2014, AJ, 148, 75

Dunlop J. S., Peacock J. A., 1993, MNRAS, 263, 936

Fanaroff B. L., Riley J. M., 1974, MNRAS, 167, 31

Fanti R., Fanti C., Schilizzi R. T., Spencer R. E., Nan Rendong, P. P., van Breugel W. J. M., Venturi T., 1990, A\&A, 231, 333

Fanti C., Fanti R., Dallacasa D., Schilizzi R. T., Spencer R. E., Stanghellini C., 1995, A\&A, 302, 317 
Gawronski M. P., Marecki A., Kunert-Bajraszewska M., Kus A. J., 2006, A\&A, 447, 63

Gendre M. A., Best P. N., Wall J. V., 2010, MNRAS, 404, 1719

Gendre M. A., Best P. N., Wall J. V., Ker L. M., 2013, MNRAS, 430, 3086

Gopal-Krishna, Wiita P. J., 2000, A\&A, 363, 507

Govoni F., Falomo R., Fasano G., Scarpa R., 2000, A\&A, 353, 507

Hardcastle M. J., Evans D. A., Croston J. H., 2007, MNRAS, 376, 1849

Heckman T. M., O’Dea C. P., Baum, S. A., Laurikainen E., ApJ, 428,65

Heckman T. M., Best P. N., 2014, ARAA, 52, 589

Hill G. J., Lilly S. J., 1991, ApJ, 367, 1

Hine R. G., Longair M. S., 1979, MNRAS, 188, 111

Kaiser C. R., Alexander P., 1999, MNRAS, 302, 515

Kaiser C. R., Best P. N., 2007, MNRAS, 381, 1548

Kauffmann G., Heckman T. M., White S. D. M., et al., 2003, MNRAS, 341, 33

Kewley L. J., Groves, B., Kauffmann G., Heckman T., 2006, MNRAS, 372, 961

Kording E. G., Jester S., Fender R., 2008, MNRAS, 383, 277

Laing R. A., Jenkins C. R., Wall J. V., Unger S. W., 1994, The First Stromlo Symposium: The Physics of Active Galaxies. ASP Conference Series, 54, 201

Ledlow M. J., Owen F. N., 1996, AJ, 112, 9

Marcha M. J. M., Browne I. W. A., Jethava N., Anton S., 2005, MNRAS, 361, 469

Meliani Z., Keppens R., Sauty C., 2010, IJMPD, 19, 867

Miraghaei H., Khosroshahi H. G., Sengupta C., Raychaudhury S., Jetha N. N., Abbassi S., 2015, AJ, 150, 196

Miraghaei H., Khosroshahi H. G., Klockner H.-R., Ponman T. J., Jetha N. N., Raychaudhury S., 2014, MNRAS, 444, 651

O’Brien A. N., Tothill N. F. H., Norris R. P., Filipovic M. D., 2016, Submitted to Proceedings of Science for "The many facets of extragalactic radio surveys: towards new scientific challenges", Bologna, Italy 20-23 October 2015 (EXTRA-RADSUR2015)

O’Dea C. P., Baum S. A., 1997, AJ, 113, 148

Owen F. N., Rudnick L., 1976, ApJL, 205, L1

Perucho M., Marti J. M., Cela J. M., Hanasz M., de La Cruz R., Rubio F., 2010, A\&A, 519, 10

Porth O., Komissarov S. S., 2015, MNRAS, 452, 1089

Pracy M. B., Ching J. H. Y., Sadler E., et al., 2016, MNRAS, 460, 2

Prestage R. M., Peacock J. A., 1988, MNRAS, 230, 131

Raimann D., Storchi-Bergmann T., Quintana H., Hunstead R., Wisotzki L., 2005, MNRAS, 364, 1239

Rigby E. E., Best P. N., Snellen I. A. G., 2008, MNRAS, 385, 310

Rigby E. E., Best P. N., Brookes M. H., Peacock J. A., Dunlop J. S., Rottgering H. J. A., Wall J. V., Ker L., 2011, MNRAS, 416, 1900

Rudnick L., Owen F. N., 1976, ApJL, 203, L107

Sabater J., Best P. N., Argudo-Fernandez M., 2013, MNRAS, 430, 638

Sadler E. M., Cannon R. D., Mauch T., et al., 2007, MNRAS, 381, 211

Sadler E. M., Ekers R. D., Mahony E. K., Mauch T., Murphy T., 2014, MNRAS, 438, 796

Scarpa, R., Urry C. M., 2001, ApJ, 556, 749

Schoenmakers A. P., de Bruyn A. G., Rottgering H. J. A., van der Laan H., Kaiser C. R., 2000, MNRAS, 315, 371

Smith E. P., Heckman T. M., 1989, ApJ, 341, 658
Strauss M. A., Weinberg D. H., Lupton R. H., et al., 2002, AJ, 124,1810

Tago E., Saar E., Tempel E., Einasto J., Einasto M., Nurmi P., Heinamaki P., 2010, A\&A, 514, 11

Tchekhovskoy A., Bromberg O., 2016, MNRAS Letters, 6

Turner R. J., Shabala S. S., 2015, ApJ, 806, 59

Wold M., Lacy M., Armus L., 2007, A\&A, 470, 531

Wykes S., Hardcastle M. J., Karakas A. I., Vink J. S., 2015, MNRAS, 447, 1001

York D. G., Adelman J., Anderson J. E., et al., 2000, AJ, 120, 1579

Yuan F., Narayan R., 2014, ARA\&A, 52, 529

Zirbel E. L., Baum S. A., 1995, ApJ, 448, 521 